\title{
A numerical study on the limitations of modal Iwan models for impulsive excitations
}

\author{
Robert M. Lacayo ${ }^{\mathrm{a}} *$, Brandon J. Deaner ${ }^{\mathrm{b}}$, Matthew S. Allen ${ }^{\mathrm{a}}$
}

\begin{abstract}
Structures with mechanical joints are difficult to model accurately. Even if the natural frequencies of the system remain essentially constant, the damping introduced by the joints is often observed to change dramatically with amplitude. Although models for individual joints have been employed with some success, accurately modeling a structure with many joints remains a significant obstacle. To this end, Segalman proposed a modal Iwan model, which simplifies the analysis by modeling a system with a linear superposition of weakly-nonlinear, uncoupled single degree-of-freedom systems or modes. Given a simulation model with discrete joints, one can identify the model for each mode by selectively exciting each mode one at a time and observing how the transient response decays. However, in the environment of interest several modes may be excited simultaneously, such as in an experiment when an impulse is applied at a discrete point. In this work, the modal Iwan model framework is assessed numerically to understand how well it captures the dynamic response of typical structures with joints when they are excited with impulsive forces applied at point locations. This is done by comparing the effective natural frequency and modal damping of the uncoupled modal models with those of truth models that include nonlinear modal coupling. These concepts are explored for two structures, a simple spring-mass system and a finite element model of a beam, both of which contain physical Iwan elements to model joint nonlinearity. The results show that modal Iwan models can effectively capture the variations in frequency and damping with amplitude, which, for damping, can increase by as much as two orders of magnitude in the microslip regime. However, even in the microslip regime the accuracy of a modal Iwan model is found to depend on whether the mode in question is dominant in the response; in some cases the effective damping that the uncoupled model predicts is found to be in error by tens of percent. Nonetheless, the modal model captures the response qualitatively and is still far superior to a linear model.
\end{abstract}

Keywords: nonlinear damping, Masing model, Hilbert transform, friction, reduced order model 


\section{Introduction}

Mechanical joints are known to be a source of considerable energy dissipation in built-up structures $[1,2]$. The presence of a joint causes the damping in the structure to show amplitudedependent behavior [3]. That is, the apparent damping in the structure tends to increase as the excitation force increases. For years, analysts have modeled amplitude-dependent damping by tuning linear equations of motion for particular levels of excitation. In realistic settings, this method can give erroneous results because the excitations from the environment induce structural responses that cover a spectrum of amplitude levels in a short time frame. A better model is one that can account for the change in damping with amplitude.

One way to account for the change in damping is simply to represent the joint as a local nonlinearity in an otherwise linear finite element model of the structure. Ideally, the joint model would be predictive, describing the friction contact in detail and capturing deformations near the interface due to the clamping load [4-6]. Prior work has shown that, at small amplitudes, the edges of the contact begin to slide relative to one another even though most of the joint remains intact $[7,8]$. This phenomenon is called microslip. In the microslip regime the stiffness of the joint decreases only slightly, but small slip displacements occur in localized regions in the contact patch that cause significant energy loss [9]. As the excitation amplitude increases, the small slip regions expand and combine until macroslip occurs, where the stiffness of the joint is compromised and relative motion occurs between the interfacing bodies.

To model microslip-macroslip phenomena in detail is exceedingly expensive, and there is still considerable uncertainty regarding which friction models should be used for a given material interface. One alternative is to replace the contacting interfaces with a lumped, hysteretic model such as an Iwan element [10]. This work uses Segalman's four-parameter Iwan model [11], which is capable of capturing joint behavior over both the microslip and macroslip regimes. Segalman's model was developed as a result of an extensive testing campaign and theoretical studies [12], and was shown to capture the physics that were observed in metal-metal contacts. A brief theoretical treatment of the four-parameter Iwan model is given in Appendix A. The fourparameter Iwan model is not predictive, however, so experiments must be conducted to derive its parameters based on the geometry, clamping force, friction characteristics, and other properties of the joint it represents. For bolted structures containing many joints, this could be a challenging task.

In response to this problem, Segalman proposed an alternative approach, the modal Iwan model, which accounts for the net effect of all the joints on each mode of the structure [13]. In Segalman's formulation, a nonlinear single-degree-of-freedom oscillator is used to represent each modal coordinate independently. The nonlinearity is captured with a four-parameter Iwan model so that each mode can exhibit both microslip and macroslip behavior. Many other researchers have proposed similar approaches, although with very different models for each mode (see for example $[14,15]$ ).

The key assumption made in these formulations is that nonlinear coupling between the linear modes is negligible, so the nonlinear model for each mode depends only on the native modal coordinate. Eriten et al. [15] used a complexification and averaging method to show rigorously that this assumption holds when the nonlinearity is weak and that the natural frequencies are not close nor integer related. Follow-up works by Deaner et al. [16] and Roettgen and Allen [17] 

free response of bolted structures in microslip.

When a single mode is selectively excited (by adjusting the spatial distribution of the force or exciting at a frequency near resonance), the coupling between modes will be relatively small. Segalman used a three-degree-of-freedom mass-spring system with a single Iwan joint to show that, when a mode is selectively excited, an appropriately tuned modal Iwan model can reproduce the modal response of that mode exceptionally well [13]. Segalman did not probe the limits of this assumption, however. When a general input is applied, modal coupling may be more important. Festjens, Chavalliear, and Dion began to explore this in [18], where they showed a case in which a model with uncoupled modes exhibited considerable error compared with their truth simulation. However, their study used an "Iwan joint" with only three slider elements in parallel, so it was not capable of accurately reproducing microslip behavior. This prompts the question: is modal coupling in realistic structures typically small enough to allow a modal Iwan model to be accurate over a significant range of amplitudes in the microslip regime?

This paper seeks to answer that question, exploring the extent to which a modal Iwan model can reproduce the response of a structure to discrete, impulsive excitations. Two structures are considered, a three-degree-of-freedom spring-mass system and a finite element model of a bolted beam, both of which contain discrete Iwan elements to describe joint behavior. The response of these structures is computed for various types of impulsive excitations, and then the effective natural frequency and damping of each mode is compared to that predicted by an uncoupled modal model. The following section presents an overview of the modal Iwan model, followed by a discussion of how to deduce its parameters based on transient ring-down data. The two structures are then studied, first by selectively exciting the linear modes of each structure to deduce the parameters of an equivalent modal Iwan model. Then the effective damping and natural frequency of the modal Iwan model are compared against those that are observed when the original finite element models are subjected to impulsive excitations at various points. The results show that the modal framework can be a good approximation in the microslip region, and they highlight the path-dependent nature of the nonlinear joints.

\section{Theoretical Background}

The objectives of this study necessitate a comparison between the response simulated from a modal Iwan model and that of a realistic system that has discrete joints. In this work, the response due to bolted-joint nonlinearity is interpreted on a mode-by-mode basis, including the modal coupling that is observed. The typical effect that bolted joints have on a structure is such that, as the amplitude of excitation increases, the modal damping of the structure increases significantly and natural frequencies decrease slightly [3]. Therefore, in addition to presenting a theoretical treatment of the modal Iwan model, this section also explains how to measure the instantaneous damping and natural frequency from a free-response signal.

\subsection{Overview of the Modal Iwan Model}

\subsubsection{Modal Equations of Motion for Structures with Joints}

Consider a finite element model of a structure whose equations of motion are 

and $\ddot{\mathbf{u}}$ are the displacement, velocity, and acceleration vectors, respectively. The vector $\mathbf{F}_{\text {ext }}$ refers to all time-dependent external loads, and $\mathbf{F}_{J}$ is the vector of joint forces due to all discrete joint elements. Here it is assumed that the constitutive models for the joint elements depend only on displacements. A Taylor series expansion about unloaded equilibrium $(\mathbf{u}=\mathbf{0})$ is applied on $\mathbf{F}_{J}$,

$$
\mathbf{F}_{J}(\mathbf{u})=\mathbf{F}_{J}(\mathbf{0})+\nabla \mathbf{F}_{J}(\mathbf{0}) \mathbf{u}+\mathbf{F}_{N L}(\mathbf{u}),
$$

where $\nabla \mathbf{F}_{J}$ is the matrix of partial derivatives of $\mathbf{F}_{J}$ with respect to each entry in $\mathbf{u}$, and $\mathbf{F}_{N L}$ is the vector of higher-order, nonlinear terms in the Taylor series. At unloaded equilibrium, the joint elements do not exert forces on the system (i.e. pre-stress is ignored and so $\left.\mathbf{F}_{J}(\mathbf{0})=\mathbf{0}\right)$, but the full stiffness of each element remains intact. Therefore, $\nabla \mathbf{F}_{J}(\mathbf{0})=\mathbf{K}_{T}$ is the stiffness matrix composed of only the tangent stiffness at equilibrium from each joint element in the system. Equation (11) becomes

$$
\mathbf{M u ̈}+\mathbf{C} \dot{\mathbf{u}}+\left(\mathbf{K}_{\infty}+\mathbf{K}_{T}\right) \mathbf{u}+\mathbf{F}_{N L}(\mathbf{u})=\mathbf{F}_{\mathrm{ext}} .
$$

Physically, $\mathbf{K}_{\infty}$ represents the stiffness of the structure at large displacement amplitudes, where the effective stiffness of all joint elements is zero (due to macroslip). This is in contrast to the limiting case where the amplitude approaches zero and the tangent stiffness of all discrete joint elements remain intact. A low-amplitude stiffness matrix can be formed as $\mathbf{K}_{0}=\mathbf{K}_{\infty}+\mathbf{K}_{T}$ to represent an equivalent linear system where all joint elements are replaced with linear springs having the same stiffness as the tangent stiffness of replaced element.

Using these stiffness matrices, the high-amplitude mode shape matrix $\Phi_{\infty}$, and the lowamplitude mode shape matrix $\boldsymbol{\Phi}_{0}$ are formed from the set of mass-normalized mode shapes, $\boldsymbol{\varphi}_{\infty r}$ and $\boldsymbol{\varphi}_{0 r}$, respectively, computed from the Eigenvalue problems

$$
\left(\mathbf{K}_{\infty}-\omega_{\infty r r}^{2} \mathbf{M}\right) \boldsymbol{\varphi}_{\infty r}=\mathbf{0}, \quad\left(\mathbf{K}_{0}-\omega_{0 r}^{2} \mathbf{M}\right) \boldsymbol{\varphi}_{0 r}=\mathbf{0},
$$

where $\omega_{\infty r r}$ and $\omega_{0 r}$ are the slip and stick natural frequencies, respectively. The modal transformation $\mathbf{u}=\boldsymbol{\Phi}_{0} \mathbf{q}$ is applied to Eq. (3) to establish an equation of motion for each modal coordinate,

$$
\ddot{q}_{r}+2 \zeta_{0 r} \omega_{0 r} \dot{q}_{r}+\omega_{0 r}^{2} q_{r}+\boldsymbol{\varphi}_{0 r}^{\mathrm{T}} \mathbf{F}_{N L}\left(\boldsymbol{\Phi}_{0} \mathbf{q}\right)=\boldsymbol{\varphi}_{0 r}^{\mathrm{T}} \mathbf{F}_{\mathrm{ext}},
$$

where $q_{r}, \dot{q}_{r}$, and $\ddot{q}_{r}$ are the modal displacement, velocity, and acceleration coordinates, respectively, governing the $r^{\text {th }}$ mass-normalized mode shape. Here it is assumed that the modal transformation diagonalizes the linear damping matrix, with $\zeta_{0 r}$ as the low-amplitude critical damping ratio for the $r^{\text {th }}$ mode. The quantity $\boldsymbol{\varphi}_{0 r}^{\mathrm{T}} \mathbf{F}_{N L}$ in Eq. (5) is a projection of nonlinear joint forces onto the $r^{\text {th }}$ mode, and the joint forces are functions of all modal coordinates. 


\subsubsection{The Modal Iwan Model}

In the modal Iwan model, two simplifying assumptions are made. The first is that the modes are uncoupled. This assumption is clearly reasonable if the response is composed of a single, dominant mode. Otherwise, if the response has multiple significant modes, then [15] shows that these modes will not interact significantly if their natural frequencies are not close or are not harmonics of other modes. With negligible modal coupling $\boldsymbol{\varphi}_{0 r}^{\mathrm{T}} \mathbf{F}_{N L}$ becomes a function of the modal coordinate $q_{r}$ of interest only.

The second simplification is that the projection of nonlinear joint forces, $\boldsymbol{\varphi}_{0 r}^{\mathrm{T}} \mathbf{F}_{N L}$, can be replaced with the force from an equivalent modal four-parameter Iwan element $\hat{F}_{I}$ (see Appendix A) minus the contribution of the linear, modal tangent stiffness term, $\hat{K}_{T} q_{r}$, as from Taylor series expansion. Considering that joints have hysteretic behavior that can be modeled with a collection of sliders, then it is reasonable to approximate the projection of their hysteresis on to the $r^{\text {th }}$ mode with a tuned Iwan element. Under these two assumptions, Equation (5) simplifies to,

$$
\ddot{q}_{r}+2 \zeta_{0 r} \omega_{0 r} \dot{q}_{r}+\omega_{0 r}^{2} q_{r}+\hat{F}_{I}\left(q_{r}\right)-\hat{K}_{T} q_{r}=\boldsymbol{\varphi}_{0 r}^{\mathrm{T}} \mathbf{F}_{\mathrm{ext}} .
$$

Note that subtracting $\hat{K}_{T}$ from the low-amplitude modal stiffness, $\omega_{0 r}^{2}$, yields the high-amplitude modal stiffness, $\omega_{\infty r}^{2}$. Equation (6) finally reduces to

$$
\ddot{q}_{r}+2 \zeta_{0 r} \omega_{0 r} \dot{q}_{r}+\omega_{\infty r}^{2} q_{r}+\hat{F}_{I}\left(q_{r}\right)=\hat{F}_{r},
$$

where $\hat{F}_{r}=\boldsymbol{\varphi}_{0 r}^{\mathrm{T}} \mathbf{F}_{\text {ext }}$ is the projection of external loads on the $r^{\text {th }}$ mode.

Equation (7) is the core of the modal Iwan model. In effect, the response of a jointed structure is approximated as a superposition of uncoupled, weakly-nonlinear modal coordinates, each governed by a single-mass oscillator system, as shown in Fig. 1. The weak nonlinearity in each modal oscillator comes from a four-parameter Iwan element whose parameters $\hat{F}_{S}, \hat{K}_{T}, \hat{\chi}$, and $\hat{\beta}$ are denoted with hats $\left(^{\wedge}\right)$ to distinguish them from the parameters of the physical joints that will be used in the models presented in Sec 3. This modal Iwan element is set in parallel with a linear spring of stiffness $\hat{K}_{\infty}=\omega_{\infty r}^{2}$ and dashpot with coefficient $2 \zeta_{0 r} \omega_{0 r}$. The reader is referred to [11] for a rigorous treatment of how to calculate $\hat{F}_{I}$ for a the four-parameter Iwan model. 


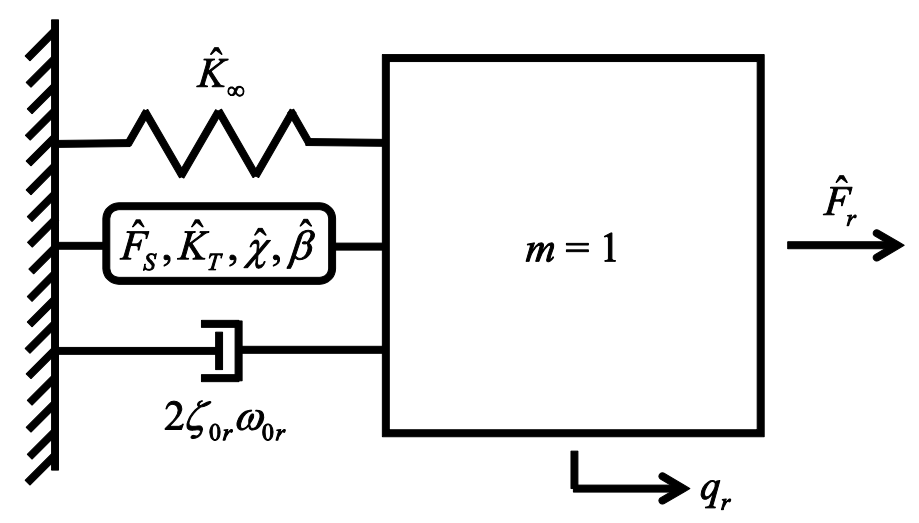

Fig. 1. Schematic of a unit mass oscillator representing a modal coordinate in a structure. The oscillator contains a four-parameter Iwan element in parallel with a linear spring and dashpot.

\subsubsection{Analytical Expressions for the Modal Damping and Natural Frequency of a Modal Iwan Model}

By assuming harmonic motion, Segalman derived analytical expressions for the Iwan element's instantaneous stiffness and energy dissipated per cycle as functions of the deflection amplitude [11]. Deaner et al. reformulated the equations to apply to a modal Iwan model, expressing them in terms of the modal displacement amplitude, $\left|q_{r}\right|$, and added contributing terms from the linear spring and damper [16]. The analytical expressions for the instantaneous modal stiffness, $\hat{K}_{r, \text { fit }}$, and dissipation, $D_{r, \text { fit }}$, associated with mode $r$ are

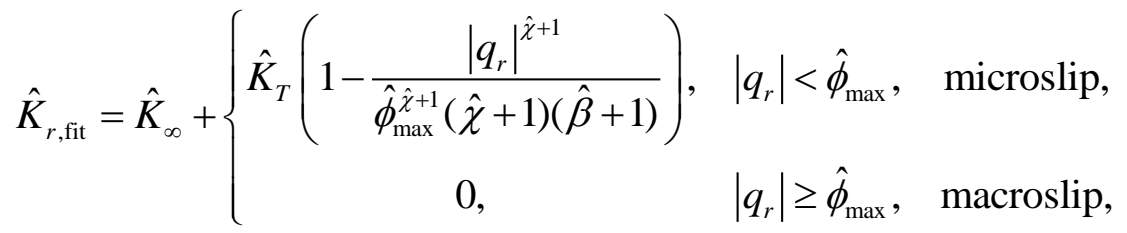

$$
\begin{aligned}
& D_{r, \mathrm{fit}}=2 \pi \zeta_{0 r} \omega_{0 r} \omega_{r, \mathrm{fit}}\left|q_{r}\right|^{2}+\left\{\begin{array}{ccc}
\frac{4 \hat{R}\left|q_{r}\right|^{\hat{\chi}+3}}{(\hat{\chi}+3)(\hat{\chi}+2)}, & \left|q_{r}\right|<\hat{\phi}_{\max }, & \text { microslip, } \\
4 \hat{F}_{S}\left|q_{r}\right|, & \left|q_{r}\right| \geq \hat{\phi}_{\max }, & \text { macroslip, }
\end{array}\right. \\
& \hat{\phi}_{\max }=\frac{\hat{F}_{S}(1+\hat{\beta})}{\hat{K}_{T}\left(\hat{\beta}+\frac{\hat{\chi}+1}{\hat{\chi}+2}\right)}, \\
& \hat{R}=\frac{\hat{F}_{S}(\hat{\chi}+1)}{\hat{\phi}_{\max }^{\chi+2}\left(\hat{\beta}+\frac{\hat{\chi}+1}{\hat{\chi}+2}\right)},
\end{aligned}
$$

where $\omega_{r, \text { fit }}$ is the instantaneous natural frequency of the mode, which is obtained from Eq. (8) through 
Following the derivation in Appendix B, a closed form expression for the instantaneous modal damping is found with Eq. (B.5) (with Eq. (B.6)),

$$
\zeta_{r, \mathrm{fit}}=\frac{D_{r, \mathrm{fit}}}{2 \pi\left(\omega_{r, \mathrm{fit}}\left|q_{r}\right|\right)^{2}} .
$$

Equations (8) and (9) - and (12) and (13) by extension - have a discontinuity at $\hat{\phi}_{\max }$, the boundary between "modal microslip" and "modal macroslip." This discontinuity is a consequence of the fact that Segalman assumed micro- and macro- slip in order to derive these closed form expressions. In contrast, when the modal model in Eq. (7) is integrated it will show a free response that transitions smoothly from macroslip into microslip, as will be shown later (see, for example, Fig. (4)). Due to the discontinuity, Eq. (12) and (13) are not used to represent the modal Iwan model in the comparison with the discrete impulse measurements. Instead, the equations are used as tools to identify the six modal Iwan model parameters $\left\{\hat{K}_{\infty}, \zeta_{0 r}, \hat{F}_{S}, \hat{K}_{T}, \hat{\chi}, \hat{\beta}\right\}$ that achieve a good fit with simulated response measurements (hence the subscript "fit"). The method for extracting the instantaneous damping and natural frequency from a free response signal is discussed in Section 2.2.

\subsection{Quantifying Damping and Natural Frequency from Response Measurements}

Measurements on the transient, free response of a structure can be processed to obtain the instantaneous damping and natural frequency data needed to fit a modal Iwan model to each mode. In practice, the measurements can be obtained either from experiments on a test structure or from truth simulations on a finite element model that uses discrete joint elements (like an Iwan element). This work uses finite element models in order to rule out model uncertainty as a cause for differences between the modal Iwan model and truth simulation. The technique for processing the measurements is based on the Hilbert transform [19] and is an adaptation of the method proposed by Sumali and Kellogg [20] and further developed in [16] and [17].

\subsubsection{Hilbert Transform Method for Free-Response Signals}

Consider a set of free-response measurements, $\dot{\mathbf{u}}(t)$, obtained from a system after subjecting it to an impact excitation. Here, it is presumed that the velocity is measured, but the procedure is easily adapted for the other states. The measurements are transformed into the modal coordinates of the low-amplitude linearized system, $\dot{\mathbf{q}}(t)$, using a modal filter

$$
\dot{\mathbf{q}}(t)=\boldsymbol{\Phi}_{0}^{-1} \dot{\mathbf{u}}(t)=\boldsymbol{\Phi}_{0}^{\mathrm{T}} \mathbf{M} \dot{\mathbf{u}}(t)
$$

where $\boldsymbol{\Phi}_{0}$ is computed as in Eq. (4) by replacing the joints with linear springs.

If the modes are sufficiently uncoupled, then each modal velocity signal $\dot{q}_{r}(t)$ should contain a single, decaying harmonic. The signal is then modeled with an exponential function,

$$
\dot{q}_{r}(t)=\operatorname{Re}\left[\exp \left(\psi_{1}(t)+\mathrm{i} \psi_{2}(t)\right)\right],
$$


where $\mathrm{i}=\sqrt{-1}$, and the amplitude and phase are slowly varying functions of time governed by $\psi_{1}(t)$ and $\psi_{2}(t)$, respectively. By computing $\dot{\tilde{q}}_{r}(t)$, the Hilbert transform of $\dot{q}_{r}(t)$, Feldman [19] shows that the amplitude and phase components can be calculated as

$$
\begin{gathered}
\psi_{1}(t)=\ln \left(\sqrt{\left(\dot{q}_{r}(t)\right)^{2}+\left(\dot{\tilde{q}}_{r}(t)\right)^{2}}\right), \\
\psi_{2}(t)=\tan ^{-1}\left(\dot{\tilde{q}}_{r}(t) / \dot{q}_{r}(t)\right) .
\end{gathered}
$$

From these expressions, the amplitude envelope is

$$
\left|\dot{q}_{r}\right| \triangleq \exp \left(\psi_{1}(t)\right)
$$

the instantaneous damped natural frequency is the time derivative of the phase,

$$
\omega_{r, \text { meas }} \triangleq \frac{\mathrm{d} \psi_{2}}{\mathrm{~d} t}
$$

and the instantaneous critical damping ratio $\zeta_{r \text {,meas }}$ can be calculated from the time derivative of the amplitude component,

$$
-\omega_{n} \zeta_{r, \text { meas }} \triangleq \alpha=\frac{\mathrm{d} \psi_{1}}{\mathrm{~d} t}
$$

where $\omega_{n}$ is the undamped natural frequency. Using the substitution $\omega_{n}=\omega_{r \text {,meas }} / \sqrt{1-\zeta_{r \text {, meas }}^{2}}$ in Eq. (20), the critical damping ratio is found to be

$$
\zeta_{r, \text { meas }} \triangleq \frac{\alpha}{\sqrt{\omega_{r, \text { meas }}^{2}+\alpha^{2}}} .
$$

Equations (18) through (21) allow one to estimate the instantaneous damping and natural frequency versus modal velocity amplitude. Equations (12) and (13), however, are expressed in terms of the modal displacement amplitude. By assuming that the change in amplitude is small over one vibration cycle, Roettgen and Allen [17] derived a simple relationship between the modal displacement $\left|q_{r}\right|$, velocity $\left|\dot{q}_{r}\right|$, and acceleration $\left|\ddot{q}_{r}\right|$ amplitudes as

$$
\omega_{r, \text { meas }}\left|q_{r}\right| \cong\left|\dot{q}_{r}\right| \cong \frac{\left|\ddot{q}_{r}\right|}{\omega_{r, \text { meas }}} .
$$

By utilizing Eq. (18), (19), and (21), each $\left|q_{r}\right|$ can be paired with a corresponding $\omega_{r \text {,meas }}$ and $\zeta_{r \text {,meas }}$ occurring simultaneously at time $t$. This establishes a relationship between amplitude and the measured natural frequency and damping that can be compared with Eq. (12) and (13). 


\subsubsection{Reducing Distortion in Signals Processed with the Hilbert Transform}

The Hilbert transform of a finite, discrete signal is susceptible to noise as well as end effects at the beginning and tail ends of a finite time signal, which very often produces $\psi_{1}(t)$ and $\psi_{2}(t)$ signals that contain spurious oscillation. The differentiation of these signals amplifies the spurious oscillation and leads to distorted curves for the instantaneous damping and frequency. In [16], [17], and [20] these oscillations were diminished by fitting polynomial functions to $\psi_{1}(t)$ and $\psi_{2}(t)$ via least-squares regression. In the authors' attempt to fit polynomials for the systems examined in this work, however, low-order polynomials do a somewhat unsatisfactory job of capturing the shape of $\psi_{1}(t)$ and $\psi_{2}(t)$, and higher-order polynomials also tend to exhibit spurious oscillations that are difficult to distinguish from the true underlying function.

For this work, the signals were instead fit with piecewise-linear functions. Such a function is as simple to fit as a low-order polynomial, yet it can follow any curve provided that a sufficiently fine mesh of points is used. The downside, of course, is that the derivative is a step function, which gives the instantaneous frequency and damping curves the appearance of being discontinuous. To address this, the derivative was computed only at the midpoint of each piecewise-linear region.

To illustrate this fitting technique, Fig. 2 shows the damping and natural frequency estimated from the transient response of the Sumali beam that will be discussed later (simulation 5 from Table D.1). The signal shown in Fig. 2(a) is the absolute value of the modal velocity for mode 7 (first bending mode), which was obtained from simulation via Eq. (14). After computing $\psi_{1}(t)$ and $\psi_{2}(t)$ using Eq. (16) and (17), the piecewise linear functions were fit to these signals using the efficient least-squares algorithm discussed in Allen et al. [21]. The fitting was done after truncating the signals to exclude the beginning and end of the time record so that the end effects did not distort the fitted function. The derivatives of the fitted curves (via Eqs. (19), (20), and (21)) led to the natural frequency and damping curves that are step functions as seen in Figs. 2(b) and 2(c). Only the midpoint values on each of these step functions were retained for further processing. 


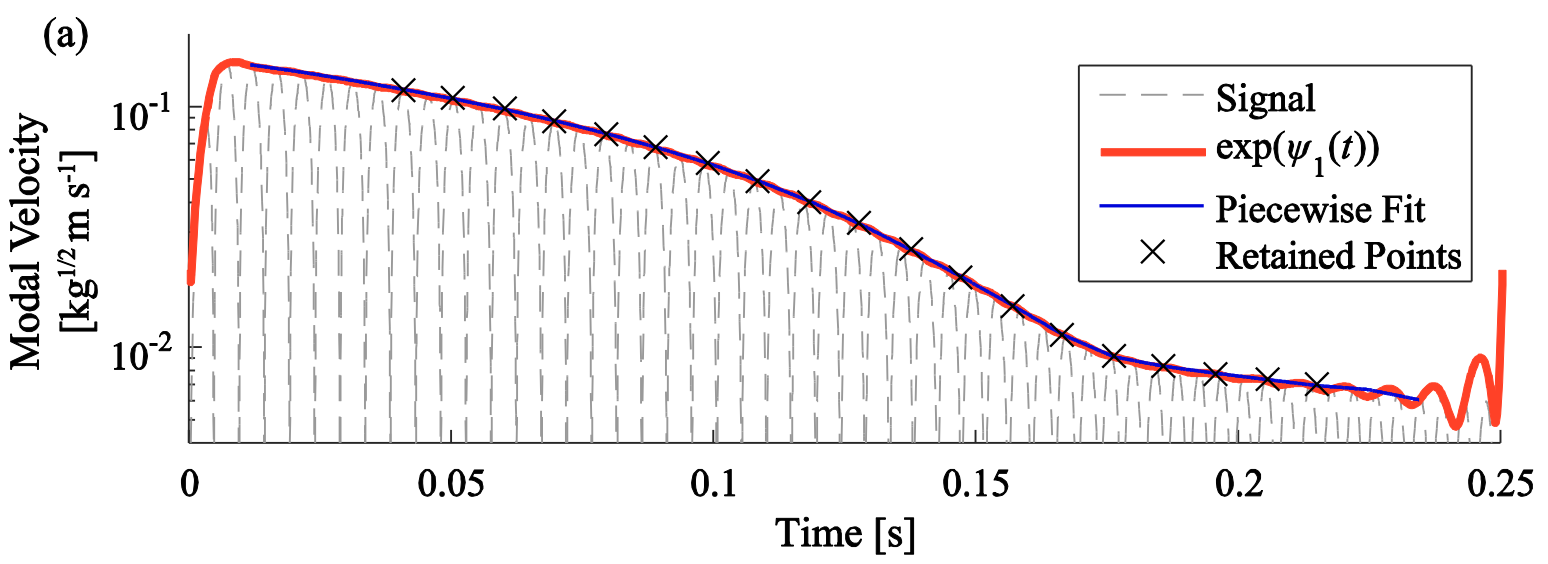

(b)

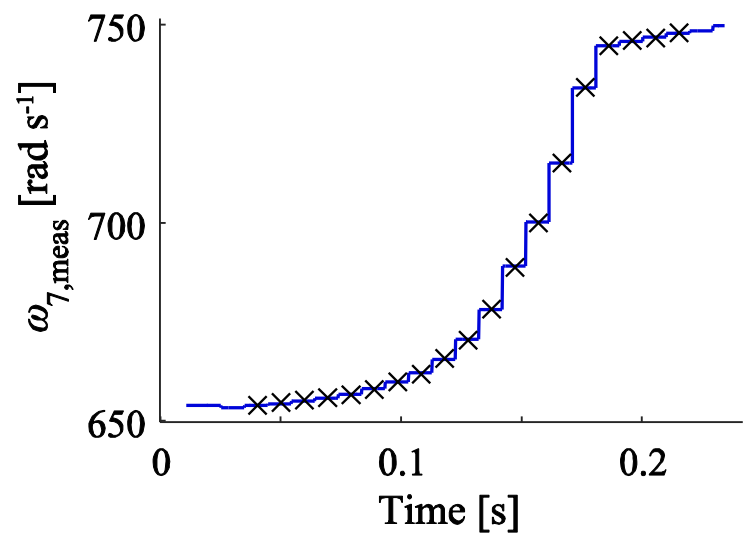

(c)

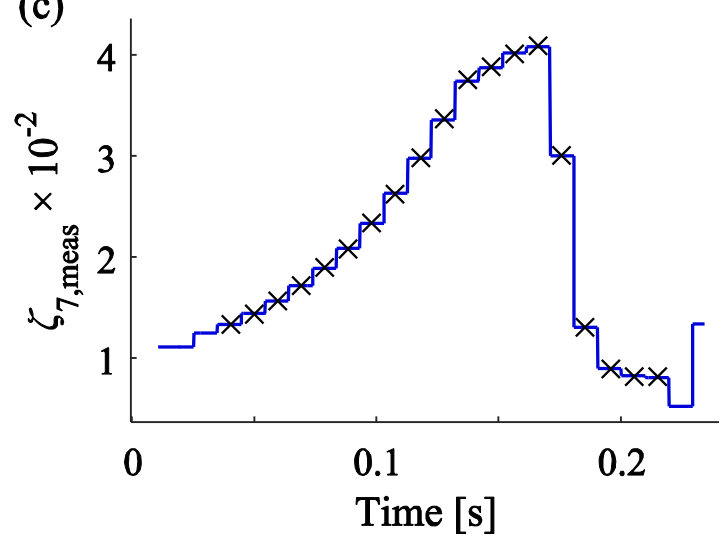

Fig. 2. (a) A response signal with Hilbert amplitude envelope, piecewise linear fit, and retained points, and the corresponding (b) instantaneous natural frequency and (c) instantaneous damping ratio. The data come from the response of simulation 5 from Table D.1, which excited mode 7 in the Sumali beam.

\subsection{Procedure for Comparison between the Modal Iwan Model and Truth Simulations}

The analysis on each of the two systems studied in this work was divided into two parts. The first part focused on fitting a modal Iwan model to each mode in the system. This part was itself divided into several steps:

Procedure I

a) An impact force was applied, distributed over the whole system as $\mathbf{F}_{\text {ext }}=\mathbf{M} \boldsymbol{\varphi}_{0 r}$, so that only the mode of interest was excited. The response to this impact was simulated until the amplitude at the end of the history was negligible compared to that at the start.

b) A modal filter was applied on the free-response signal using Eq. (14) to retrieve the modal response, $q_{r}(t)$, of interest. The Hilbert analysis described Section 2.2 was performed on the modal signal to collect $\omega_{r \text {,meas }}, \zeta_{r \text {, meas }}$ and $\left|q_{r}\right|$. 
c) Steps (a) and (b) were repeated for different forcing levels, and $\omega_{r \text {,meas }}$ and $\zeta_{r \text {,meas }}$ were compiled for a wide, logarithmic range of $\left|q_{r}\right|$ spanning the microslip and macroslip regimes.

d) Equations (12) and (13) $\left(\omega_{r \text {,fit }}\right.$ and $\left.\zeta_{r \text {,fit }}\right)$ were fit over the compiled $\omega_{r \text {,meas }}$ and $\zeta_{r \text {,meas }}$ by choice of the six modal Iwan model parameters $\left\{\hat{K}_{\infty}, \zeta_{0 r}, \hat{F}_{S}, \hat{K}_{T}, \hat{\chi}, \hat{\beta}\right\}$.

e) All steps above were repeated for each mode of interest.

Regarding step (d), the method of fitting used in this work follows a graphical approach similar to that used by Deaner et al. whereby initial guesses were made for the six modal Iwan model parameters based on the shape of the $\omega_{r \text {,meas }}$ versus $\left|q_{r}\right|$ and $\zeta_{r \text {,meas }}$ versus $\left|q_{r}\right|$ curves [16]. The parameters were then adjusted iteratively until the same curves for Eq. (12) and (13) aligned well with the measured curves. The final values for the six parameters were then associated with the modal Iwan model for that mode.

The second part of the analysis arranged the comparison between the modal Iwan models (as tuned from part 1) and the truth measurements for the amplitude-dependent damping and natural frequency. This part required processing not only the response of the system when subjected to discrete impacts, but also the simulated response of the modal Iwan model. The damping and natural frequency for the modal Iwan model was obtained as follows:

\section{Procedure II}

f) Equation (7) was integrated numerically using the tuned parameters to simulate the free-response decay of the mode of interest to a modal impulse excitation.

g) The Hilbert analysis described in Section 2.2 was performed on the modal freeresponse signal. The instantaneous natural frequency and damping obtained from Eq. (19) and (21), respectively, were denoted as $\omega_{r, M}$ and $\zeta_{r, M}$ to distinguish them as modal Iwan model properties.

h) Steps (f) and (g) were repeated for multiple excitation levels to compile $\omega_{r, M}$ and $\zeta_{r, M}$ for a logarithmic range of $\left|q_{r}\right|$. These steps were again repeated for every mode with tuned parameters.

The $\omega_{r, M}$ and $\zeta_{r, M}$ versus $\left|q_{r}\right|$ curves were compared against those obtained from the response of discrete impact excitations, which followed similar steps:

Procedure III

i) The system was excited with an impact applied at a single point on the structure, and the free-response decay signal was collected.

j) Equation (14) was applied to filter the signal into its component modes. The signals from all modes of interest were kept since a discrete impact excited more than one mode. The Hilbert analysis of Section 2.2 was performed to obtain $\omega_{r \text {,meas }}$ and $\zeta_{r \text {,meas }}$ 
for all modes of interest (i.e. this was performed on each linear modal coordinate, since each mode could be excited by the discrete impact).

k) Steps (i) and (j) were repeated for multiple excitation levels, and $\omega_{r \text {,meas }}$ and $\zeta_{r \text {,meas }}$ were compiled for each mode.

1) Steps (i) to (k) were repeated for impacts at different discrete locations on the system.

Since different points on a structure give different spatial contributions to each mode, $\omega_{r \text {,meas }}$ and $\zeta_{r \text {,meas }}$ were measured for different impact locations to investigate their range of scatter relative to $\omega_{r, M}$ and $\zeta_{r, M}$.

\section{Numerical Simulation Test Models}

The previous sections describe how to derive closed-form expressions for the damping and natural frequency for a modal Iwan model and fit them to the instantaneous damping and natural frequency estimated from dynamic simulations. This section demonstrates these techniques on two nonlinear models - a three-mass system and a two-beam finite element model - in order to assess the extent to which a modal Iwan model can reproduce the response of these systems.

\subsection{Three-Mass Discrete System}

The first structural model was the same three-degree-of-freedom academic model investigated by Segalman [13]. The three-mass model, as seen in Fig. 3, consisted of three identical masses, $m=10$, connected with linear springs of stiffness $k=9$. A discrete fourparameter Iwan element was placed between the second and third masses with parameters $F_{S}=10, K_{T}=1, \chi=-0.5$, and $\beta=5$.

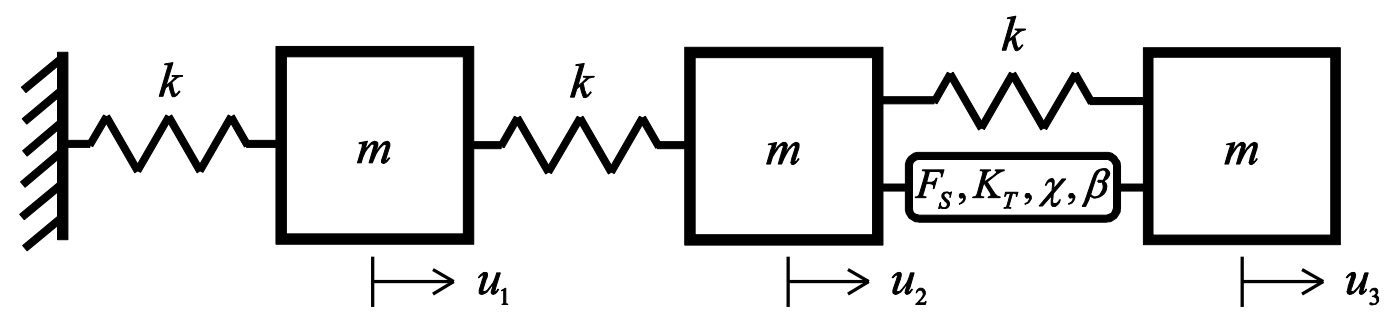

Fig. 3. Schematic of the three-mass system with a discrete Iwan joint model connecting the second and third masses.

The dynamics of this system were modeled using Eq. (1). The mass matrix for the three-mass system was defined as

$$
\mathbf{M}=\left[\begin{array}{ccc}
m & 0 & 0 \\
0 & m & 0 \\
0 & 0 & m
\end{array}\right],
$$

and the stiffness matrices were 
The low amplitude mass-normalized mode shapes $\boldsymbol{\varphi}_{0 r}$ were computed with Eq. (4) and are shown in Table 1. In observing the relative displacements between the second and third masses, it is apparent that in mode 1 the Iwan joint sees almost an order of magnitude less deflection than for mode 2 or mode 3 . Therefore mode 1 was expected to exercise the Iwan joint less than mode 2 and mode 3 , leading to less damping at the same magnitude.

\section{Table 1}

Mass-normalized mode shapes for the three-mass system.

\begin{tabular}{cccc}
\hline DOF & $\boldsymbol{\varphi}_{01}$ & $\boldsymbol{\varphi}_{02}$ & $\boldsymbol{\varphi}_{03}$ \\
& $\left(\omega_{01}=0.425\right)$ & $\left(\omega_{02}=1.216\right)$ & $\left(\omega_{03}=1.744\right)$ \\
\hline$u_{1}$ & 0.1051 & 0.2433 & -0.1725 \\
$u_{2}$ & 0.1891 & 0.0871 & 0.2380 \\
$u_{3}$ & 0.2307 & -0.1822 & -0.1166 \\
\hline
\end{tabular}

Each mode of the three-mass system was assigned a linear critical damping ratio of $\zeta_{0 r}=1 \times 10^{-4}$. The damping matrix, $\mathbf{C}$, was then defined as

$$
\mathbf{C}=\mathbf{M} \boldsymbol{\Phi}_{0} \operatorname{diag}\left[2 \omega_{0 r} \zeta_{0 r}\right] \boldsymbol{\Phi}_{0}^{\mathrm{T}} \mathbf{M},
$$

with diag $\left[2 \omega_{0 r} \zeta_{0 r}\right]$ denoting a diagonal matrix where the $r^{\text {th }}$ diagonal entry contains the value $2 \omega_{0 r} \zeta_{0 r}$. Lastly, the joint force vector was defined as

$$
\mathbf{F}_{J}=\left\{\begin{array}{c}
0 \\
-F_{I} \\
F_{I}
\end{array}\right\},
$$

where $F_{I}$ was the force exerted on the system by the discrete Iwan element (see Appendix A), which depended on the relative displacement $u_{3}-u_{2}$.

\subsubsection{Tuning Modal Iwan Models to the Three-Mass System}

The first set of simulations for the three-mass system selectively excited each of the three modes to parameterize a modal Iwan model for each mode. To excite a single mode, $\mathbf{F}_{\text {ext }}$ in Eq. (1) was defined as follows,

$$
\mathbf{F}_{\text {ext }}(t)=p \mathbf{M} \varphi \sin \left(\omega_{0 r} t\right), \quad 0 \leq t<\pi / \omega_{0 r},
$$


where $p$ was a scalar multiplier used to adjust the force magnitude, and the mode shape vector $\boldsymbol{\varphi}$ was a placeholder for either $\boldsymbol{\varphi}_{0 r}$ or $\boldsymbol{\varphi}_{\infty r}$. When completing the simulations, $\boldsymbol{\varphi}_{0 r}$ tended to give cleaner modal coordinate signals (not contaminated by signals from other modes) when the system was excited in microslip, whereas $\boldsymbol{\varphi}_{\infty r}$ gave cleaner signals for macroslip excitations. Therefore the two vectors were interchanged depending on the value of $p$. The values of $p$ and the corresponding $\varphi$ used in these simulations are detailed in Table 2. Equation (1) was integrated numerically in MATLAB using an iterative, average-acceleration Newmark method [22] with a time step of $2 \times 10^{-2}$ for 200000 steps for all simulations in this system.

Table 2

The $p$ values and $\varphi$ vectors used in the simulations that excite each mode of the three-mass system.

\begin{tabular}{|c|c|c|c|c|c|c|c|c|}
\hline \multicolumn{3}{|c|}{ Mode 1} & \multicolumn{3}{|c|}{ Mode 2} & \multicolumn{3}{|c|}{ Mode 3} \\
\hline Sim No. & $p$ & $\varphi$ & Sim No. & $p$ & $\varphi$ & Sim No. & $p$ & $\varphi$ \\
\hline 1 & 6000 & $\boldsymbol{\varphi}_{\infty 1}$ & 10 & 50000 & $\boldsymbol{\varphi}_{\infty 2}$ & 19 & 200000 & $\boldsymbol{\varphi}_{\infty 03}$ \\
\hline 2 & 3000 & $\boldsymbol{\varphi}_{\infty 1}$ & 11 & 10000 & $\boldsymbol{\varphi}_{\infty 2}$ & 20 & 75000 & $\boldsymbol{\varphi}_{\infty 03}$ \\
\hline 3 & 1000 & $\boldsymbol{\varphi}_{\infty 1}$ & 12 & 3400 & $\boldsymbol{\varphi}_{\infty 2}$ & 21 & 15000 & $\boldsymbol{\varphi}_{\infty 33}$ \\
\hline 4 & 450 & $\boldsymbol{\varphi}_{\infty 1}$ & 13 & 200 & $\boldsymbol{\varphi}_{\infty 2}$ & 22 & 5600 & $\boldsymbol{\varphi}_{\infty 03}$ \\
\hline 5 & 150 & $\boldsymbol{\varphi}_{\infty 1}$ & 14 & 9 & $\boldsymbol{\varphi}_{02}$ & 23 & 150 & $\boldsymbol{\varphi}_{\infty 03}$ \\
\hline 6 & 33 & $\boldsymbol{\varphi}_{01}$ & 15 & 3 & $\boldsymbol{\varphi}_{02}$ & 24 & 12 & $\varphi_{03}$ \\
\hline 7 & 10 & $\boldsymbol{\varphi}_{01}$ & 16 & 0.8 & $\varphi_{02}$ & 25 & 2 & $\varphi_{03}$ \\
\hline 8 & 3.4 & $\varphi_{01}$ & 17 & 0.2 & $\boldsymbol{\varphi}_{02}$ & 26 & 0.38 & $\varphi_{03}$ \\
\hline 9 & 1 & $\boldsymbol{\varphi}_{01}$ & 18 & 0.04 & $\boldsymbol{\varphi}_{02}$ & 27 & 0.096 & $\boldsymbol{\varphi}_{03}$ \\
\hline- & - & - & - & - & - & 28 & 0.192 & $\varphi_{03}$ \\
\hline
\end{tabular}

Table 3 lists the modal Iwan model parameters that were estimated for each mode by following Procedure I in Section 2.3. Note that for all three modes, $\zeta_{0}=1 \times 10^{-4}$ is the same value that was prescribed for linear damping in Eq. (25). This makes sense because Eq. (5) establishes that, in the limit that displacement amplitude goes to zero, the system approaches the linear extreme in which the equivalent modal damping is equal to $\zeta_{0 r}$. In other words, $\zeta_{r \text {,meas }}$ asymptotes to $\zeta_{0}$ at low amplitudes. 


\section{Table 3}

Fitted modal Iwan parameters for three-mass system.

\begin{tabular}{cccc}
\hline Parameter & Mode 1 & Mode 2 & Mode 3 \\
\hline$\hat{K}_{\infty}$ & 0.1782 & 1.399 & 2.922 \\
$\zeta_{0 r}$ & $1.0 \times 10^{-4}$ & $1.0 \times 10^{-4}$ & $1.0 \times 10^{-4}$ \\
$\hat{F}_{S}$ & 0.4462 & 2.877 & 3.278 \\
$\hat{K}_{T}$ & $1.917 \times 10^{-3}$ & $7.843 \times 10^{-2}$ & 0.1196 \\
$\hat{\chi}$ & -0.5002 & -0.5150 & -0.5070 \\
$\hat{\beta}$ & 5.700 & 5.614 & 4.800 \\
\hline
\end{tabular}

Figure 4 plots the compiled $\omega_{2 \text {,meas }}$ and $\zeta_{2 \text {,meas }}$ versus $\left|q_{2}\right|$ data for mode 2 obtained from the simulations of Table 2. The data are juxtaposed with the fitted $\omega_{2, \text { fit }}$ and $\zeta_{2, \text { fit }}$ curves produced from the parameters listed in Table 3 and using the closed form expressions in Eqs. (12) and (13). Here it can be seen that the fitted curves follow the measured data very well in the microslip regime and in the late macroslip regime, but the jump discontinuity that occurs at the threshold of macroslip in Eq. (8) and (9) creates a region of error in the early macroslip regime. This is simply an approximation error in the closed form expressions and can be avoided by simply simulating the response of the 1DOF modal Iwan model as explained below rather than relying on the closed form expressions. The frequency and damping versus amplitude plots for the first and third modes look similar to those in Fig. 4, and are shown in Fig. C.1 in Appendix C.

Time domain simulations on the modal Iwan model were conducted for all three modes by following Procedure II using the parameters listed in Table 3. The modal force in Eq. (7) was defined as

$$
\hat{F}_{r}(t)=p \sin \left(\omega_{0 r} t\right), \quad 0 \leq t<\pi / \omega_{0 r} .
$$

The same number of simulations as listed in Table 2 were conducted using the same $p$ values. The simulations were completed by numerically integrating Eq. (7) in MATLAB using the Newmark method with a time step of $2 \times 10^{-2}$ for 400000 steps. The compiled $\omega_{2, M}$ and $\zeta_{2, M}$ versus $\left|q_{2}\right|$ curves from the mode 2 simulations are plotted with the measured data and fitted curves in Fig. 4. With selective modal excitation, one can observe that the modal Iwan model fits the measured response of each mode very well all regimes of slip. The same conclusion can be drawn from the $\omega_{r, M}$ and $\zeta_{r, M}$ curves for modes 1 and 3 in Fig. C.1. 


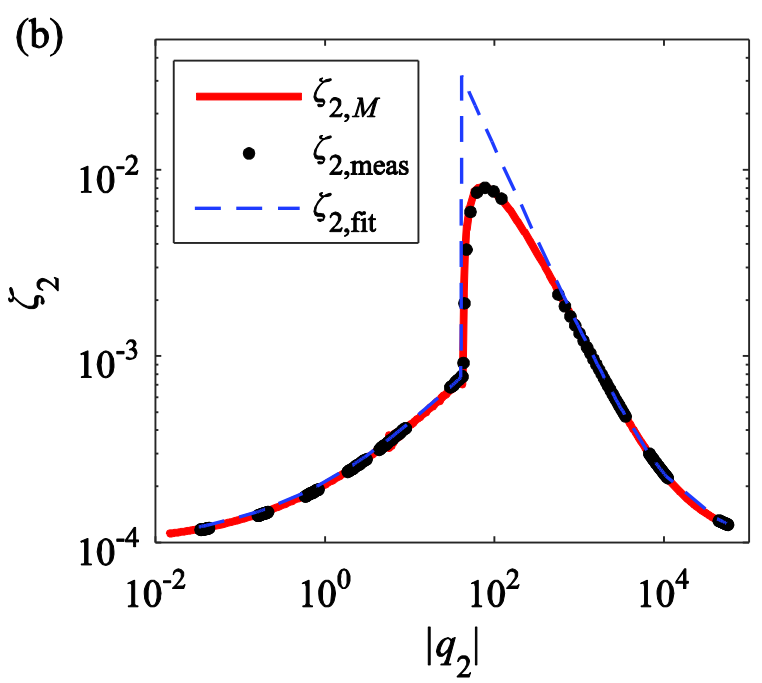

Fig. 4. (a) Natural frequency and (b) critical damping ratio versus modal displacement amplitude when the $2^{\text {nd }}$ mode of the three-mass system is selectively excited.

Of particular note for the second and third modes is that the damping in the microslip regime increases to more than six times the low-amplitude damping as the macroslip regime is approached. Clearly, to model this system as linear with only the low-amplitude damping would result in a stark underestimation of the damping. The first mode, on the other hand, only shows a doubling of damping in the microslip regime, but this might be expected since the first mode shape barely exercises the Iwan element.

\subsubsection{Discrete Impact Simulations on the Three-Mass System}

The $\omega_{r, M}$ and $\zeta_{r, M}$ curves computed in Section 3.1.1 for the three-mass system were then tested against the measured damping and natural frequency produced from impact excitations on each mass individually. To retrieve the data, Eq. (1) was again integrated numerically, but the external force was computed as

$$
\mathbf{F}_{\text {ext }}(t)=p \mathbf{P} \sin \left(\omega_{03} t\right), \quad 0 \leq t<\pi / \omega_{03},
$$

where $\mathbf{P}$ is a vector of zeros except for a single 1 at the entry corresponding to the mass where excitation is desired. Nine impact simulations were conducted on each mass for $p$ equal to $150000,50000,15000,5000,500,30,10,3$ and 1 . The same integrator settings used for the mode excitation simulations were also used for these simulations. Figures 5, 6, and 7 show the $\omega_{r \text {,meas }}$ and $\zeta_{r \text {,meas }}$ versus $\left|q_{r}\right|$ curves for the first, second, and third modes, respectively, as computed using Procedure III. 

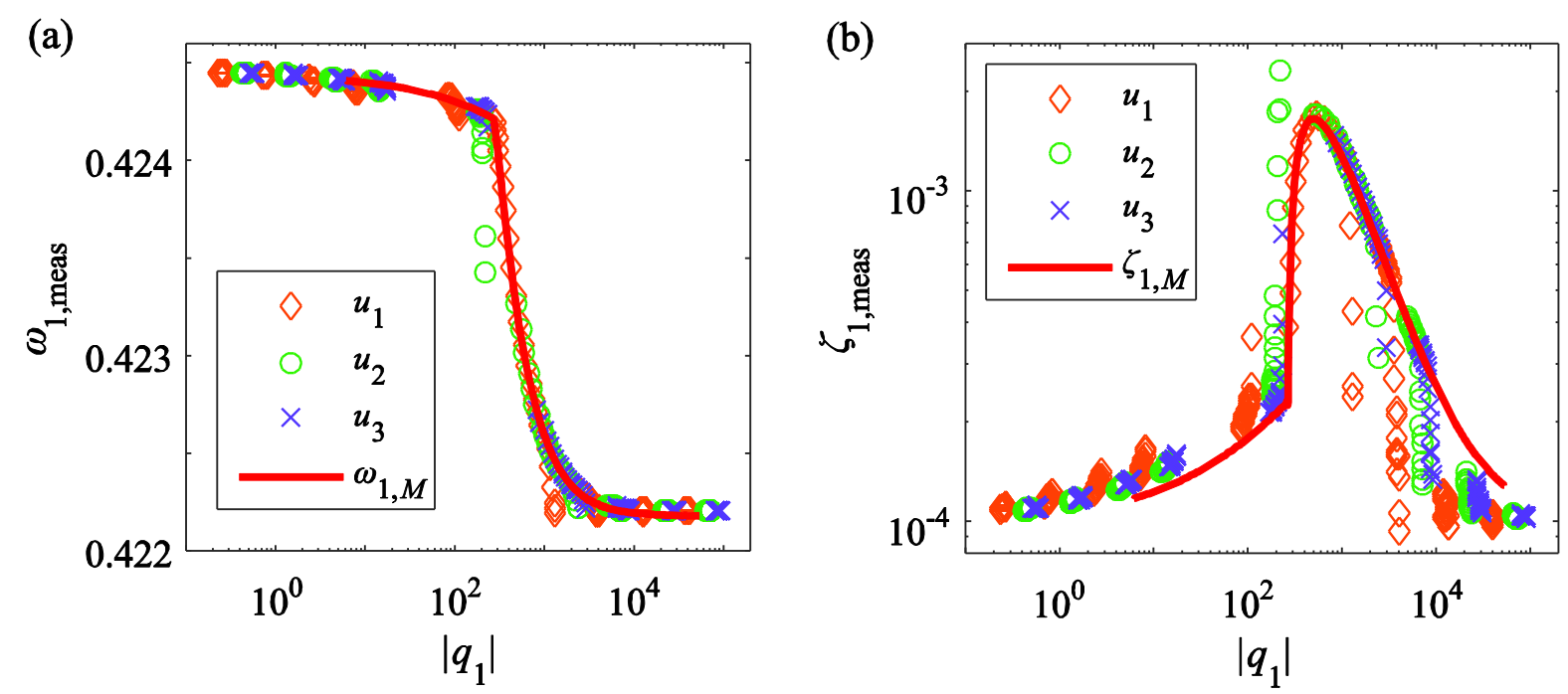

Fig. 5. The measured (a) Natural frequency and (b) critical damping ratio versus modal displacement amplitude for the mode 1 response to discrete impulses in the three-mass system. Points denoted with the same marker originated from a simulation where the mass noted in the legend was excited. The $\omega_{r, M}$ and $\zeta_{r, M}$ from the modal Iwan model is overlaid for comparison.

(a)

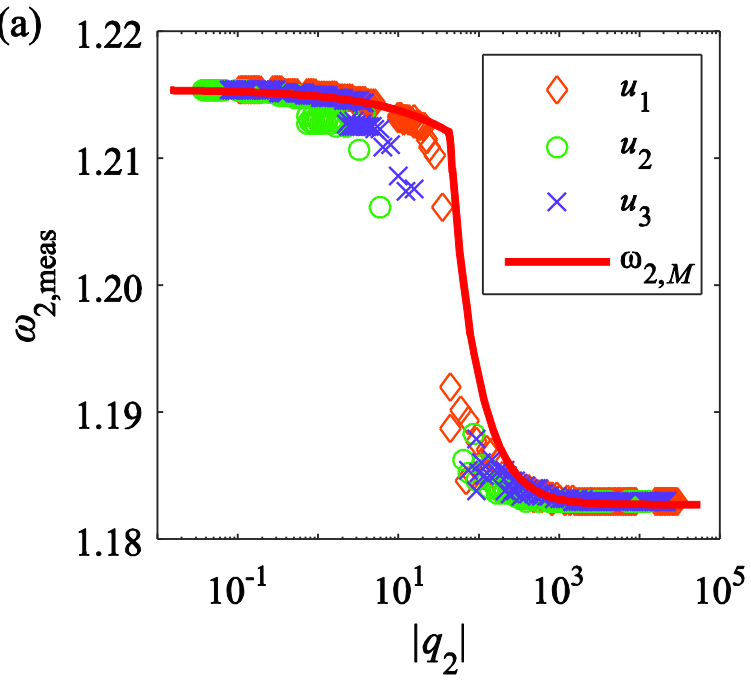

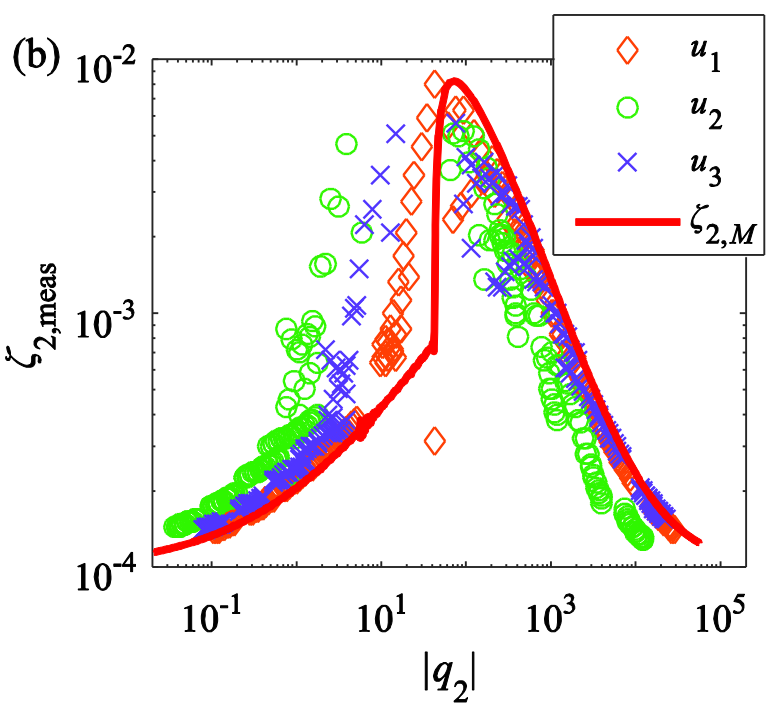

Fig. 6. The measured (a) Natural frequency and (b) critical damping ratio versus modal displacement amplitude for the mode 2 response to discrete impulses in the three-mass system. 
(a)

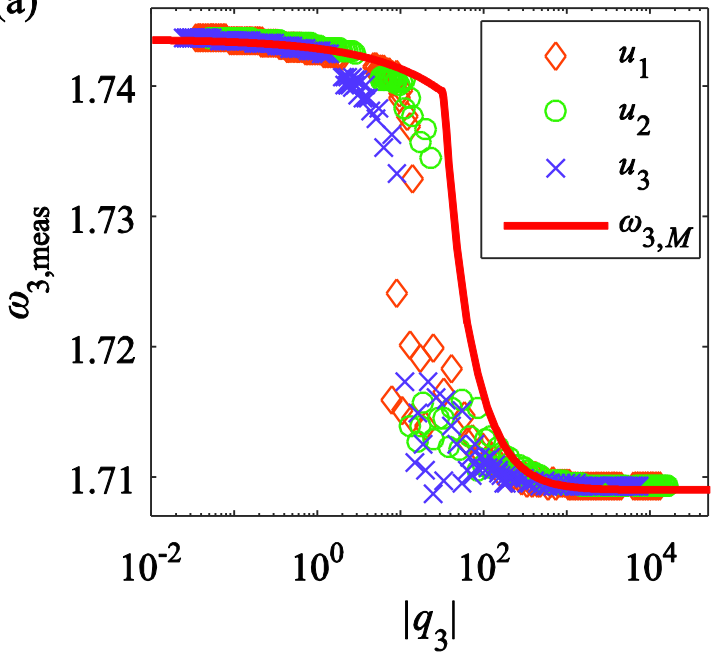

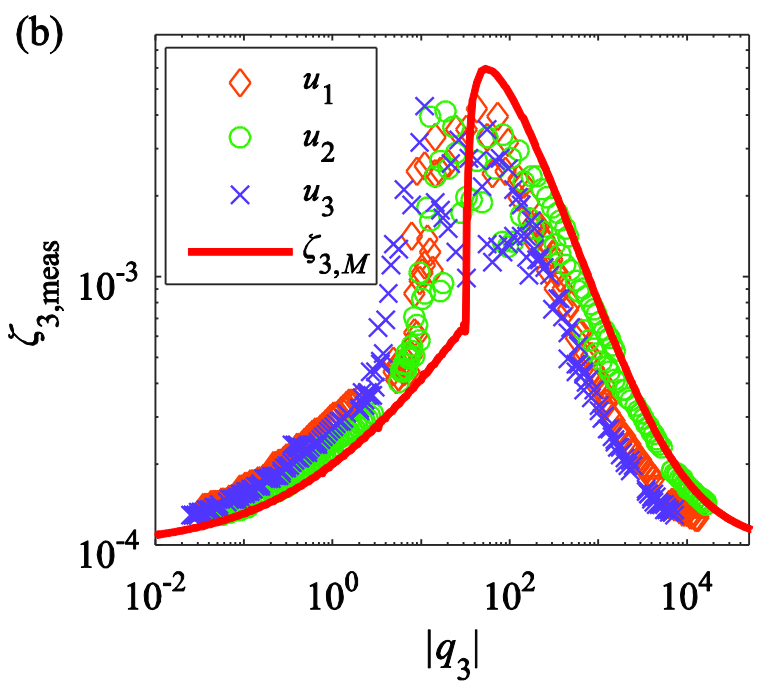

Fig. 7. The measured (a) Natural frequency and (b) critical damping ratio versus modal displacement amplitude for the mode 3 response to discrete impulses in the three-mass system.

\subsubsection{Comparison and Results Discussion}

The results in Fig. 6 show that the modal Iwan model does a good job of predicting the effective natural frequency and damping of the system, although there are discrepancies and they can be significant in some cases. The micro-slip regime (far left in each plot) is considered first as this is the regime in which joints are typically designed to function and also the regime in which the modal approach is expected to be reasonably accurate.

While the modal Iwan model is qualitatively correct in the microslip regime, it does significantly underestimate the damping in several cases. In general, the largest error in damping seems to occur when a mode a mode is weak at the excitation point. This makes sense, because the response to an impulse at that point will be dominated by a different mode, so the singlemode approximation for the weak mode becomes less accurate. For example, the second mode for the three-mass system is very weak when mass $u_{2}$ is excited, and the modal Iwan model underestimates damping by as much as $67 \%$ in the microslip in that case. However, with that same excitation the first and third modes are more strongly excited, so the modal Iwan models for these modes were more accurate, underestimating damping by no more than $13 \%$ for the first mode and $17 \%$ for the third mode.

Considering now the entire plots including the macroslip regime, the modal Iwan model seems to underestimate microslip damping and overestimate macroslip damping in all impulse simulations. Most striking for the second and third modes is that the modal Iwan model vastly underpredicts (by a whole order of magnitude) the simulations that occur in the early macroslip regime and transition into the microslip regime.

Quantitatively, the cause for this apparent shift in the damping curves may be accounted for by considering nonlinear coupling between modes. Recall that the formulation for the modal Iwan model is described fundamentally by Eq. (7), where the modal Iwan force $\hat{F}_{I}$ is assumed to be a function of the native modal coordinate only. This is a simplification of $\boldsymbol{\varphi}_{0 r}^{\mathrm{T}} \mathbf{F}_{J}$, which is a 
function of all modal degrees of freedom. For the three-mass system, the exact projection of joint forces onto a particular mode $r$ is actually

$$
\boldsymbol{\varphi}_{0 r}^{\mathrm{T}} \mathbf{F}_{J}(\mathbf{u})=\boldsymbol{\varphi}_{0 r}^{\mathrm{T}} \mathbf{F}_{J}\left(\boldsymbol{\varphi}_{01} q_{1}+\boldsymbol{\varphi}_{02} q_{2}+\boldsymbol{\varphi}_{03} q_{3}\right)
$$

When the second mode is selectively excited (as in Fig. (4)), then $q_{2}$ is dominant in $\mathbf{F}_{J}$ and the modal Iwan assumption is reasonable. On the other hand, a force at a single point excites all modes at once. As a result the state of the joint is governed by the sum of the active modal coordinates, so the damping is activated to a higher amplitude than would be expected from $q_{2}$ alone. This activation to higher amplitude is the source of the downward amplitude shift that $\omega_{r, \text { meas }}$ and $\zeta_{r \text {,meas }}$ experience relative to $\omega_{r, M}$ and $\zeta_{r, M}$, seen in Figs. 5 to 7.

Figure 8 illustrates this effect for mode 1 using the $p=15000$ simulation for excitation at mass $u_{2}$. Figure 8(a) shows that the measured damping ratio for mode 1 starts at a low value, but then rises up to match the analytical curve with only slight change in modal displacement amplitude. This phenomenon appears contradictory, but the plot does not show the response of the second and third modes. If the damping curve in Fig. 8(a) is instead shown versus time (Fig. $8(b)$ ), one notices that the damping ratio is consistent with that of the analytical fit for time $t>1700$.

Figure 8(c) shows the amplitude envelopes for the three modal coordinates versus time in this same simulation. It is clear that modes 2 and 3 are nearly as significant as mode 1 at the beginning of the time history. Therefore, at the beginning of the simulation, the joint forces $\mathbf{F}_{J}(\mathbf{u})$ produce lower damping in mode 1 than would be expected if only mode 1 were excited because the physical Iwan elements is activated to a higher amplitude in the macroslip regime. The other modes, $q_{2}$ and $q_{3}$, decay rapidly until $t>1700$, at which point they can be considered small compared to $q_{1}$. At this point, $\mathbf{F}_{J}$ can be considered to depend on $q_{1}$ only, so $\zeta_{r \text {, meas }}$ then follows $\zeta_{1, M}$ for the first mode closely. 
(a)

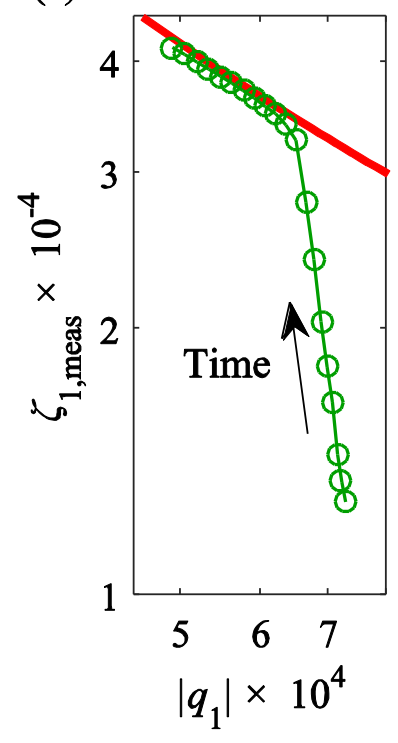

(b)

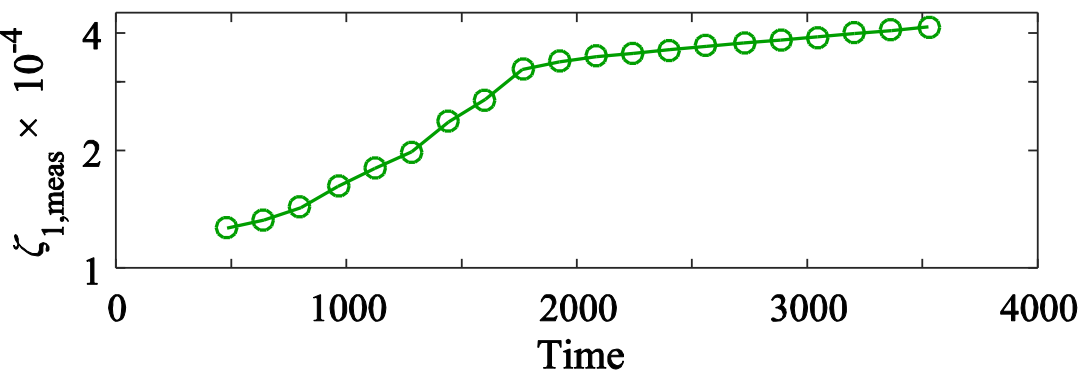

(c)

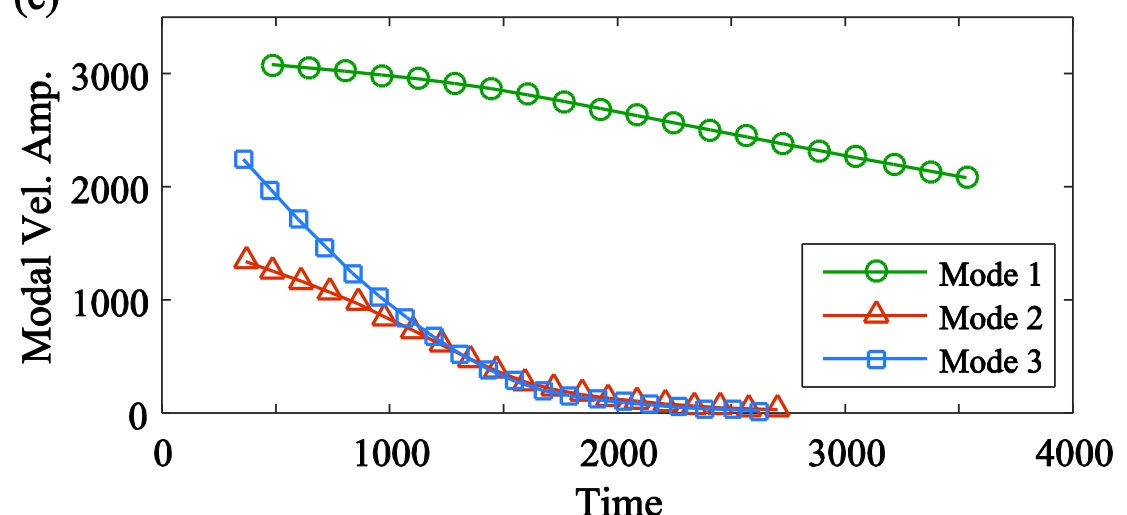

Fig. 8. (a) Damping versus displacement amplitude (shown with $\zeta_{1, M}$ as the thick line) and (b) damping versus time for Mode 1 response to an impulse on mass $u_{2}$, and (c) the modal velocity amplitude time histories for all three modes in the same simulation. The data are from the $p=$ 15000 simulation for the three-mass system. Time units are in seconds, modal displacement amplitudes are in $\mathrm{kg}^{1 / 2} \mathrm{~m}$, and modal velocity amplitude units are in $\mathrm{kg}^{1 / 2} \mathrm{~m} \mathrm{~s}^{-1}$.

In summary, the error in the modal Iwan model can be accounted for by considering the neglected modal coupling. In the truth models, the coupling causes the physical joints to be activated to a higher amplitude than would be expected from a single modal coordinate in isolation. This explains why the modal Iwan model underestimates damping in the microslip regime and overestimates damping in the macroslip regime.

Even so, the error is not bad if the impacts excite the three-mass system to amplitudes confined well within the microslip or macroslip regimes. In microslip, the error is greatest in mode 2 where $\zeta_{2, M}$ underestimates the mass 2 impact measurements by a factor of $0.6(67 \%$ underestimation) at amplitude $\left|q_{2}\right|=0.5$. The error in macroslip is greatest for mode 1 , where an excitation on mass 1 causes $\zeta_{1, M}$ to overestimate measurements at the beginning of the time signal by a factor of 5 (400\% overestimation) at $\left|q_{1}\right|=4000$. The modal signal in both of these cases, however, does not dominate the response, so although that mode's damping would not be accurately simulated, its contribution to the overall response may be neglected. For the most dominant modal signals, $\zeta_{r, M}$ underestimates the modal signals confined within microslip by an average of $5.6 \%$, and overestimates those confined in macroslip by an average of $23 \%$.

The modal Iwan model produces the greatest error in the late microslip region near the threshold for macroslip. Based on the amplitude of the native modal coordinate, the modal Iwan 
model predicts that the structure should be in "modal microslip," but in the truth models the physical joints themselves have already passed the threshold for macroslip due to the added contribution from other modes. Therefore the measured modal damping has already been pushed into the peak region of the curve, causing the modal Iwan model underestimate measured damping by a whole order of magnitude. This error must be accepted if one wishes to profit from the advantages of a modal Iwan model. However, there is consolation in the fact that joints are designed to operate in microslip. In fact, experimental studies have shown that it is notoriously difficult to impact a real, experimental structure with a hammer such that macroslip or transitioning behavior can be measured $[16,17]$. More often than not, one desires a model that describes only microslip behavior. One notable exception is the case of blast loads as experienced when the stages of launch vehicles separate, and so there is certainly interest in extending this work so that macroslip can be captured.

\subsection{Sumali Beam Finite Element Model}

To see whether these findings would hold for a more realistic structure, a finite element model of an assembly of two beams, here dubbed the Sumali beam, was also investigated. Deaner et al. studied this same structure in experiments and fitted modal Iwan models to their measurements [16], so some effort was made to tune the finite element model so that it approximated their results.

The Sumali beam structure contains two thin, identical, stainless steel beams that overlap and are joined with four bolts, as shown Fig. 9. Each beam has length $508 \mathrm{~mm}$, width $50.8 \mathrm{~mm}$, and thickness $6.35 \mathrm{~mm}$, and the area of overlap extends $355.6 \mathrm{~mm}$ along the length of each beam. The four bolts are distributed in single file along the middle axis of the beam, each separated by a uniform distance of $76.2 \mathrm{~mm}$.

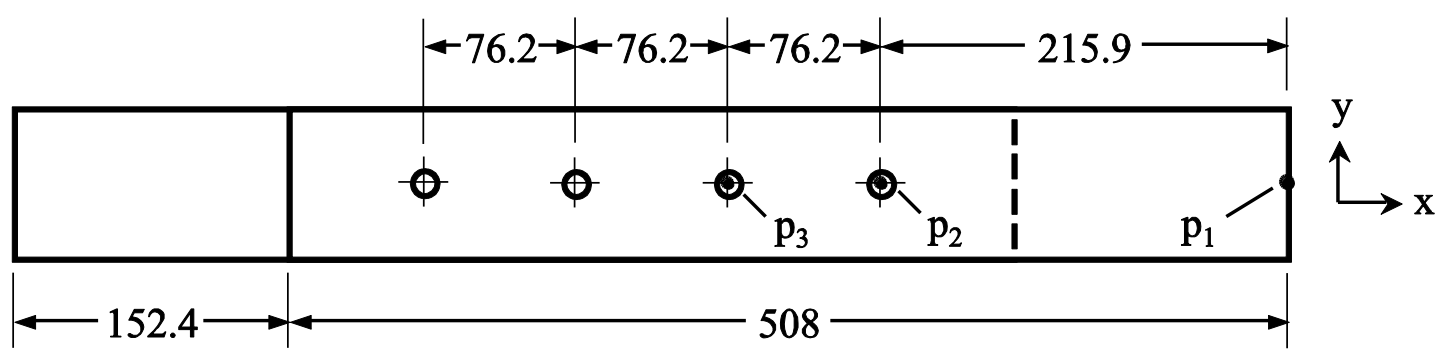

Fig. 9. Schematic of the Sumali beam with major dimensions. Length units are in millimeters. Figure is not drawn to scale.

The two beams were included in the finite element model used in this analysis. Each beam was meshed with 640 8-node hexahedron elements constructed using an isotropic, elastic material model with Young's modulus $248 \mathrm{GPa}$, Poisson's ratio 0.29, and mass density $8000 \mathrm{~kg}$ $\mathrm{m}^{-3}$. The boundary conditions were taken to be in free-free configuration to match the experimental setup.

The bolts themselves were not meshed, but were instead replaced by discrete joint models located between the beams. The forces exerted by each joint model were distributed over an area of contact surrounding the position where each bolt would be found. An illustration of each joint model is given in Fig. 10. On the two interfacing surfaces of the beams, all nodes within a 25.4 $\mathrm{mm} \times 25.4 \mathrm{~mm}$ square contact patch surrounding a bolt were tied to a virtual node using a RBE3 
element spider [23], a type of averaging multi-point constraint. This virtual node represents an average of all degrees of freedom (DOF) on the contact patch in both translation and rotation. The six DOF from one virtual node were then coupled to the six DOF from the corresponding virtual node on the opposite surface using a joint model consisting of linear springs, Iwan elements, and a rigid constraint equations, the details of which are given in Section 3.2.1.

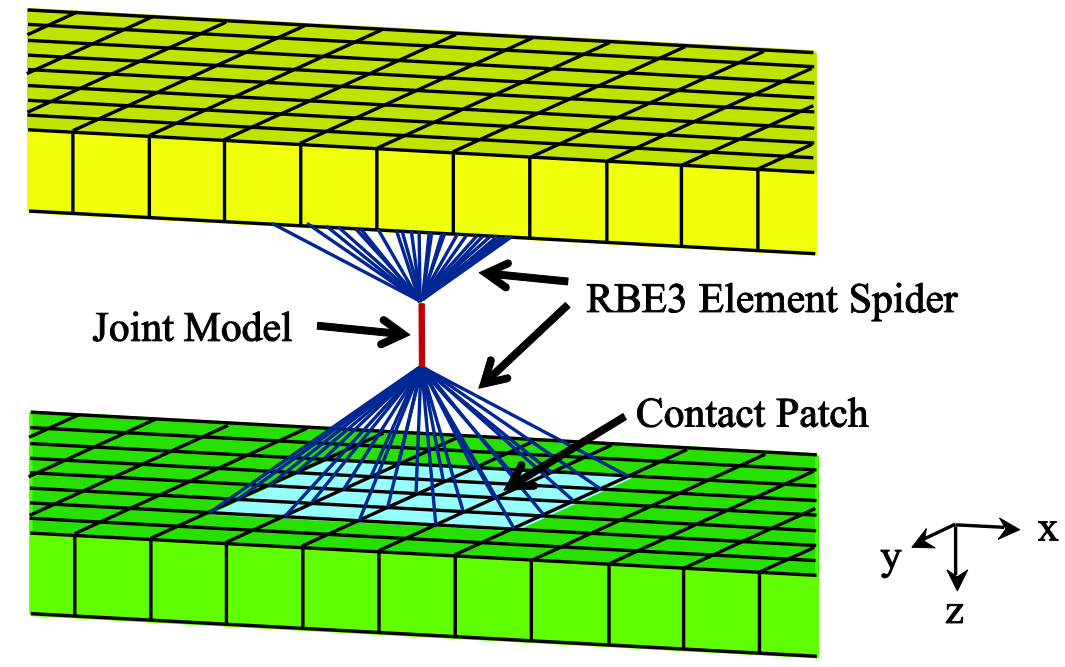

Fig. 10. Exploded view of a discrete joint model in the Sumali beam finite element model.

To speed up computation time for simulation, the mass and stiffness matrices produced from this finite element model (sans the joint models) were reduced using the Craig-Bampton reduction technique [24]. The eight joint virtual nodes (six DOF each) were retained as the interface in the Craig-Bampton model, along with point $\mathrm{p}_{1}$ (three translational DOF) shown in Fig. 9, so there were a total of 51 constraint modes. Of the resulting fixed-interface modes, only the first ten were kept to include the first torsion and the first three bending modes. The reduction was performed internally within SIERRA/SD [23], a structural dynamics solver for finite element models developed by Sandia National Laboratories. The reduced mass and stiffness matrices were then imported into MATLAB, where the joint models were added and the response of the assembly was simulated.

\subsubsection{Joint Model Characterization}

Deaner et al. [16] measured the natural frequencies and modal Iwan parameters for the first three modes of the Sumali beam at three different bolt torque values. The measurements pertaining to the torque of $3.39 \mathrm{~N} \mathrm{~m}$ were used to tune the model for this work. An independent set of parameters for each of the four joint models cannot be determined from the provided experimental data, so all joint models were simply assumed to have the same parameterization.

For simplicity, a single Iwan element was oriented in the $\mathrm{x}$-translation direction in each joint model since it was assumed that the first few bending modes exercise friction in this direction the most. In a first pass, though, only linear springs were attached to each translational and rotational DOF (except z-rotation to allow out-of-plane rotation), and the stiffness of each spring was then adjusted iteratively until the linear natural frequencies of the beam matched (as closely as possible) the low-amplitude natural frequencies measured in [16]. In this iterative process, it was found that adjusting the stiffness of the $\mathrm{z}$-translation, and $\mathrm{x}$ and $\mathrm{y}$-rotation springs did not affect 
the natural frequencies significantly. Those DOF were instead rigidly coupled using a constraint equation to further reduce the total number of DOF to 49.

As a result of the updating steps described above, the natural frequencies of the first and second bending modes matched the experimentally-derived values well (at low amplitude excitation). The stiffness of the $\mathrm{x}$-translation spring was then decreased until the linear natural frequencies closely matched the high-amplitude natural frequencies reported in [16]. The amount of decrease in stiffness of the x-translational spring became the value of the tangent stiffness parameter, $K_{T}$, for the discrete Iwan models. The other three parameters, $F_{S}, \chi$, and $\beta$, were simply taken as the geometric mean of the modal Iwan values determined for the first three bending modes [16]. While this updating procedure had not resulted in a model that rigorously matched the dynamic response that was measured, it was thought nonetheless to be a feasible truth model, and it showed ample joint energy dissipation for the study that follows.

The final elements used for all four joint models are a linear spring with stiffness $5.3 \times 10^{7}$ $\mathrm{N} \mathrm{m}^{-1}$ oriented in the $\mathrm{x}$-translation direction, a linear spring with stiffness $5.25 \times 10^{8}$ $\mathrm{N} \mathrm{m}^{-1}$ oriented in y-translation, and an Iwan element with parameters $F_{S}=30.7 \mathrm{~N}$, $K_{T}=4.73 \times 10^{8} \mathrm{~N} \mathrm{~m}^{-1}, \chi=-0.23$, and $\beta=0.46$ oriented in x-translation in parallel with the spring. The natural frequencies of the assembly can then be computed with and without the joints present, and Table 4 lists the final low and high-amplitude natural frequencies (computed from Eq. (4)) for the Sumali beam with free-free boundary conditions. The first six modes are rigid body modes, while modes 7, 8, and 9 are the first, second, and third bending modes, respectively.

\section{Table 4}

Comparison between finite element model and experiment for low-amplitude natural frequencies, $\omega_{0}$, and high-amplitude natural frequencies, $\omega_{\infty}$, of the Sumali beam.

\begin{tabular}{|c|c|c|c|c|c|c|}
\hline Mode & $\begin{array}{c}\text { Model } \\
\omega_{0}\left(\operatorname{rad~s}^{-1}\right)\end{array}$ & $\begin{array}{c}\text { Deaner [16] } \\
\omega_{0}\left(\operatorname{rad~s}^{-1}\right)\end{array}$ & Error & $\begin{array}{c}\text { Model } \\
\omega_{\infty}\left(\operatorname{rad~s}^{-1}\right)\end{array}$ & $\begin{array}{l}\text { Deaner [16] } \\
\omega_{\infty}\left(\operatorname{rad~s}^{-1}\right)\end{array}$ & Error \\
\hline 7 & 759.3 & 759.6 & $0.04 \%$ & 652.2 & 664.1 & $1.79 \%$ \\
\hline 8 & 1345 & 1350 & $0.37 \%$ & 1297 & 1145 & $13.3 \%$ \\
\hline 9 & 3185 & 2976 & $7.02 \%$ & 2713 & 2739 & $0.95 \%$ \\
\hline
\end{tabular}

\subsubsection{Tuning Modal Iwan Models to the Sumali Beam System}

As was done for the three-mass system, modal Iwan models were tuned for the Sumali beam system first by exciting the system using the force defined in Eq. (27), and the response was found by integrating Eq. (1) using a Newmark algorithm in MATLAB. The mass matrix $\mathbf{M}$ in this case was the reduced mass matrix from Craig-Bampton reduction. The stiffness matrix $\mathbf{K}_{\infty}$ was the reduced stiffness matrix that includes coupling terms from the linear springs in the joint models, but not the stiffness contribution from the Iwan elements. The damping matrix was calculated with Eq. (25), again assigning $\zeta_{0 r}=1 \times 10^{-4}$ for all modes. The forces from all Iwan elements are summed in the $\mathbf{F}_{J}$ vector while following the same sign convention used in Eq. (26). The force magnitude $p$ values and mode shape $\varphi$ vectors used for excitation are listed in Table D.1 in Appendix D. 
Procedure I was completed to retrieve $\omega_{r \text {,meas }}$ and $\zeta_{r \text {,meas }}$ for these modal excitation simulations and fit $\omega_{r \text {,fit }}$ and $\zeta_{r \text {,fit }}$ curves. The fitted modal Iwan model parameters are listed in Table 5. Then Procedure II was completed to simulate the modal Iwan model, where each simulation was performed using the model parameters listed in Table 5, the excitation given in Eq. (28), and the same integrator parameters given in Table D.1.

\section{Table 5}

Modal Iwan parameters for the Sumali beam reduced-order model.

\begin{tabular}{cccc}
\hline Parameter & Mode 7 & Mode 8 & Mode 9 \\
\hline$\hat{K}_{\infty}\left[\mathrm{s}^{-2}\right]$ & $4.235 \times 10^{5}$ & $1.673 \times 10^{6}$ & $7.321 \times 10^{6}$ \\
$\zeta_{0 r}$ & $1.0 \times 10^{-4}$ & $1.0 \times 10^{-4}$ & $1.0 \times 10^{-4}$ \\
$\hat{F}_{S}\left[\mathrm{~N} \mathrm{~kg}^{-1 / 2}\right]$ & 1.802 & 1.518 & 6.934 \\
$\hat{K}_{T}\left[\mathrm{~s}^{-2}\right]$ & $1.537 \times 10^{5}$ & $1.340 \times 10^{5}$ & $2.822 \times 10^{6}$ \\
$\hat{\chi}$ & -0.1369 & -0.1620 & -0.1008 \\
$\hat{\beta}$ & 2.561 & 2.000 & 1.529 \\
\hline
\end{tabular}

The measured, fitted, and modal Iwan frequency and damping curves for mode 8 are shown in Fig. 11. The agreement between the measured and modal Iwan curves is representative of those of mode 7 and mode 9, which are illustrated in Fig. D.1 in Appendix D. Again, the modal Iwan model reproduces the frequency and damping exceptionally well over a wide range of amplitudes. Also noted are that the damping in microslip for the second bending mode rises to a value 20 times greater than that of the low-amplitude, linear damping. For the first and third bending modes, the damping rises by a factor of almost 100 . This shows, again, how modeling the system linearly with low-amplitude damping is insufficient to capture the dynamic response for a more general range of amplitudes.
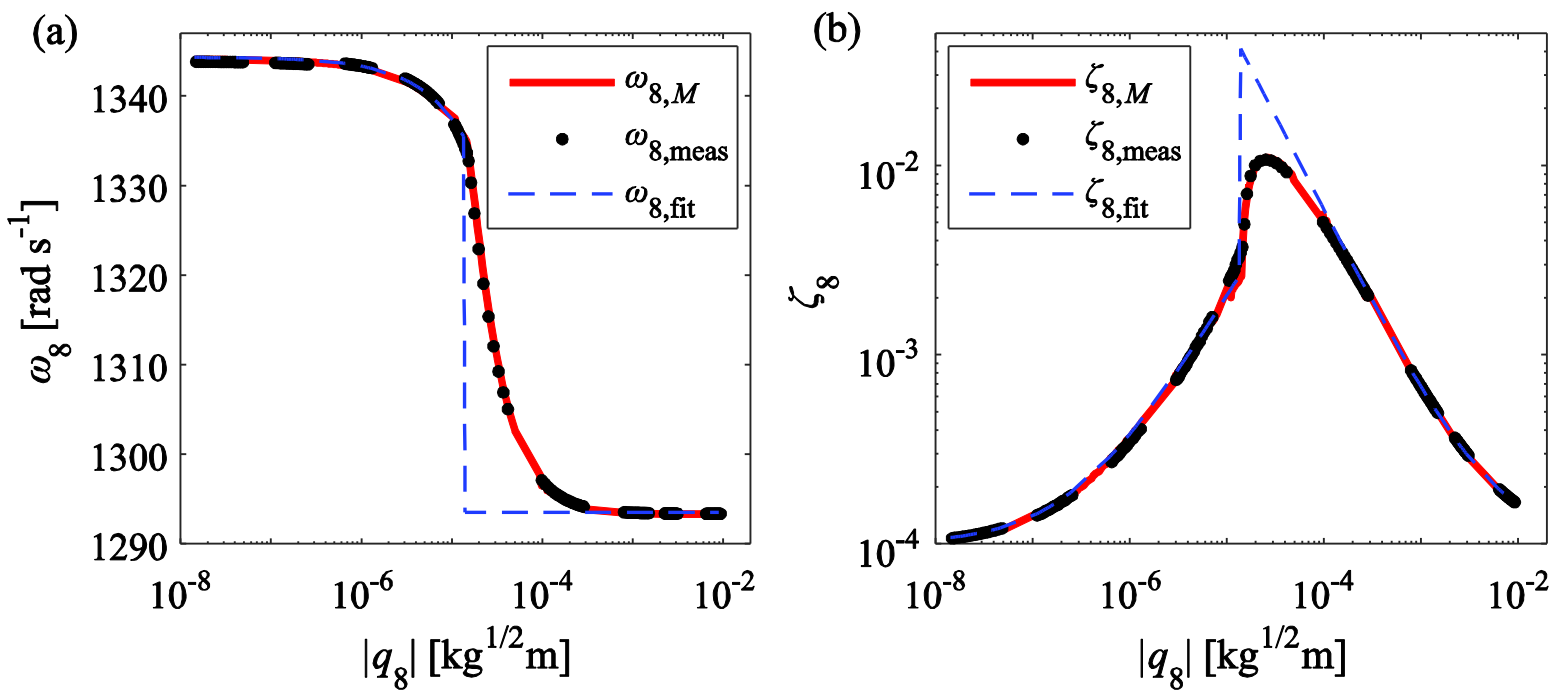

Fig. 11. (a) Natural frequency and (b) critical damping ratio versus modal displacement for selective excitation of mode 8 of the Sumali beam. 


\subsubsection{Discrete Impact Simulations on the Sumali Beam System}

Procedure III was implemented to acquire $\omega_{r \text {,meas }}$ and $\zeta_{r \text {,meas }}$ from the measured response to point impact excitations on the Sumali Beam. Three impact locations were investigated, corresponding to points $\mathrm{p}_{1}, \mathrm{p}_{2}$, and $\mathrm{p}_{3}$ shown in Fig. 9, with the force oriented along the $\mathrm{z}$ direction. Point $\mathrm{p}_{1}$ is a node located midway along the edge of the beam. Points $\mathrm{p}_{2}$ and $\mathrm{p}_{3}$ are bolt locations, but in the finite element model the force is actually applied on the coordinate governing the z-translation degree of freedom in the two virtual nodes at each point. The shape of the impact force follows Eq. (29) with the mode 9 natural frequency, $\omega_{09}$, substituted in the place of $\omega_{03}$. The force multiplier $p$ values, and other simulation parameters, are listed in Table D.2 in Appendix D. The $\omega_{r \text {,meas }}$ and $\zeta_{r \text {,meas }}$ curves for all three excitation points are compared with $\omega_{r, M}$ and $\zeta_{r, M}$ obtained in Section 3.2.2 in Fig. 12 (mode 7), Fig. 13 (mode 8), and Fig. 14 (mode 9).
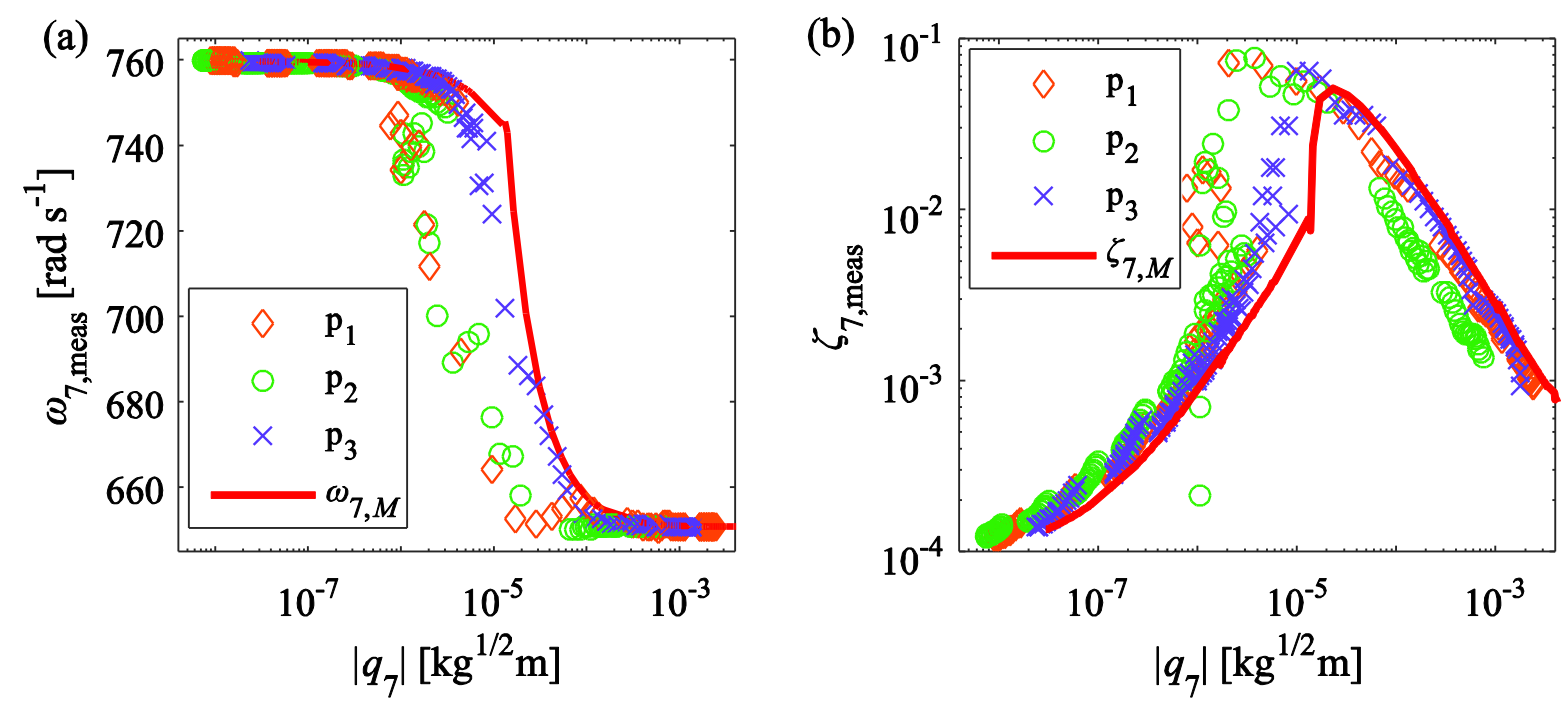

Fig. 12. The measured (a) Natural frequency and (b) critical damping ratio versus modal displacement amplitude for the mode 7 response to discrete impulses in the Sumali beam system. Points denoted with the same marker originated from a simulation where the same point was excited. The $\omega_{r, M}$ and $\zeta_{r, M}$ curves from the modal Iwan model are overlaid for comparison. 

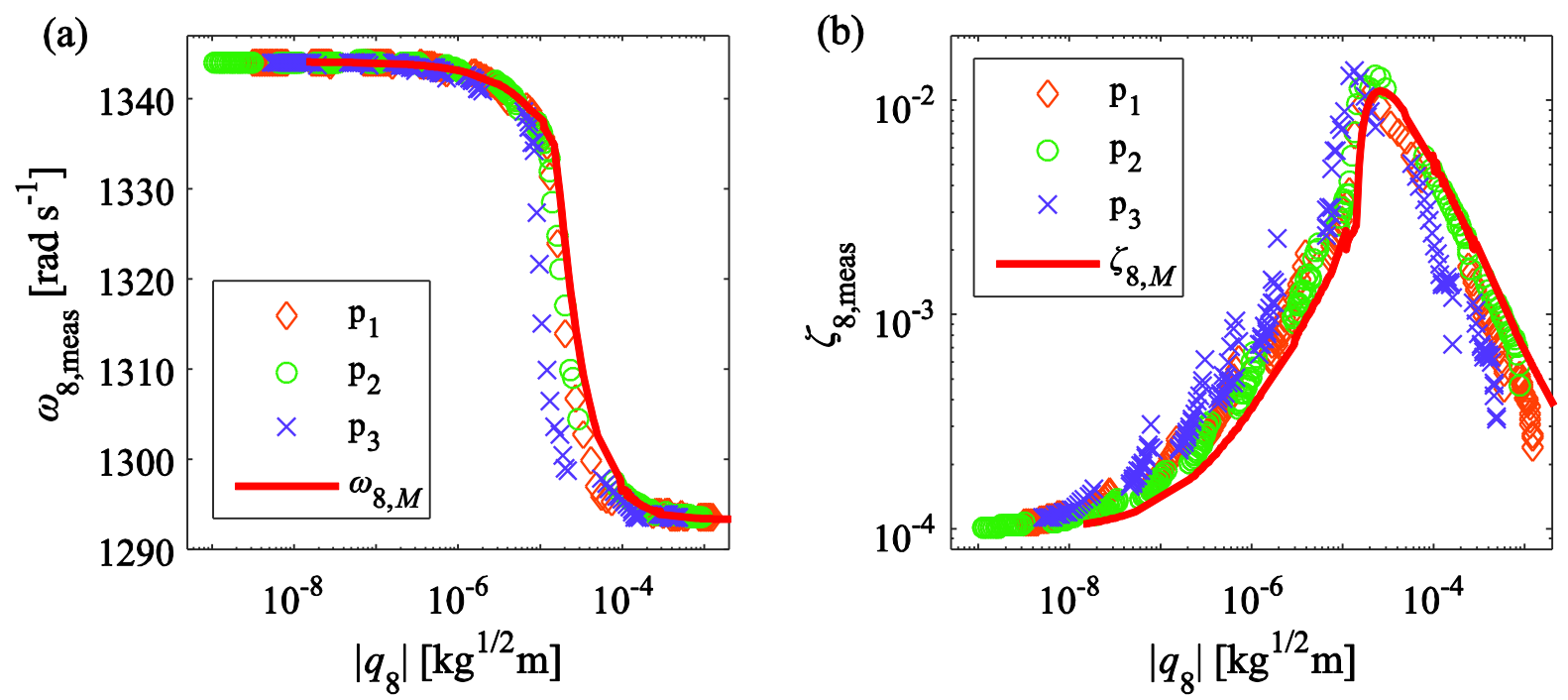

Fig. 13. The measured (a) Natural frequency and (b) critical damping ratio versus modal displacement amplitude for the mode 8 response to discrete impulses in the Sumali beam system.
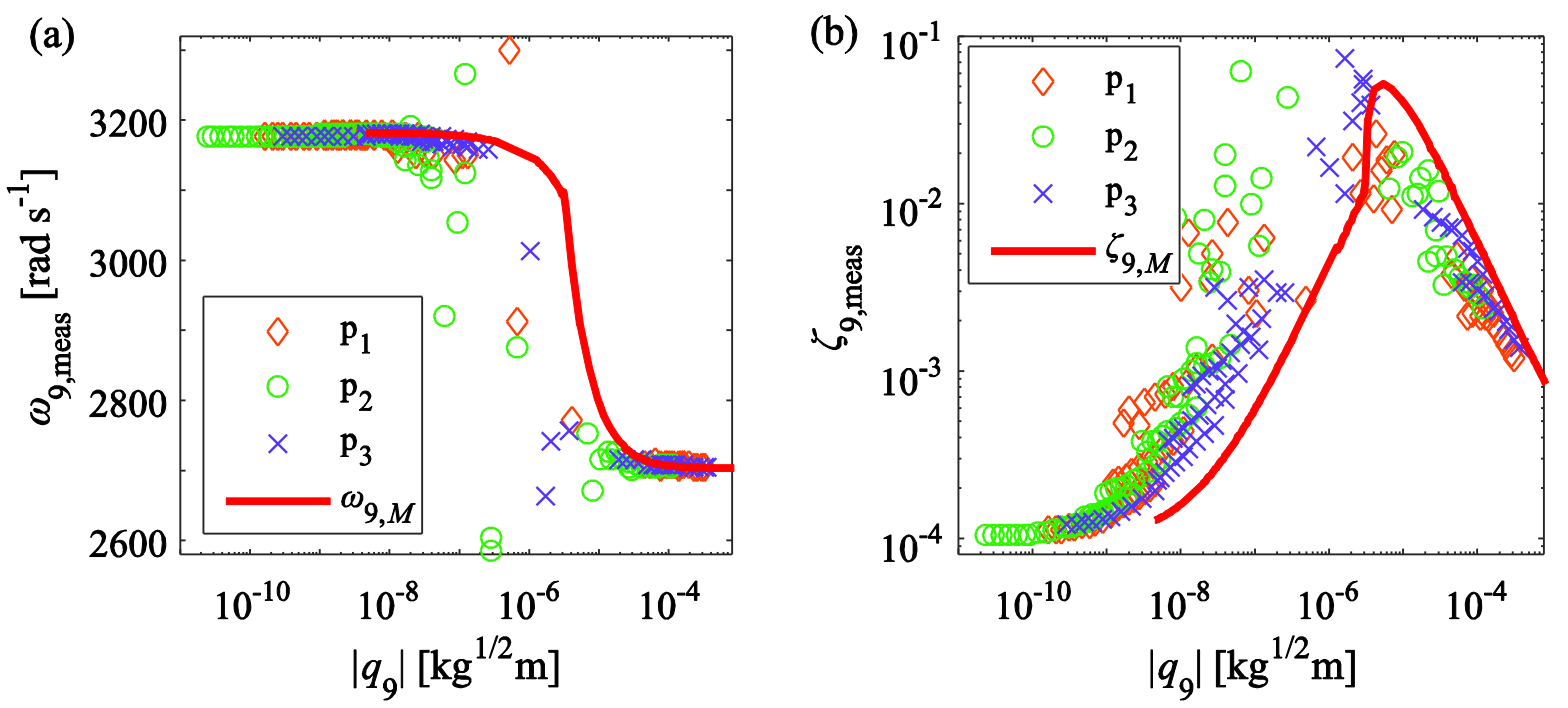

Fig. 14. The measured (a) Natural frequency and (b) critical damping ratio versus modal displacement amplitude for the mode 9 response to discrete impulses in the Sumali beam system.

\subsubsection{Sumali Beam Results Discussion}

Similar to the three-mass system, the modal Iwan models do well in maintaining the shape of the frequency and damping curves for the seventh and eighth modes. However, the models are in error for the same reasons regarding modal coupling discussed in Section 3.1.3.

In the seventh mode, for example, $\zeta_{7, M}$ underestimates the measured damping for the impulse response at point $\mathrm{p}_{2}$ (least dominant modal signal) by an average of $68 \%$ near the late 
microslip regime. This discrepancy decreases to a $44 \%$ underestimation at lower amplitudes. In mode $8, \zeta_{8, M}$ maintains an average underestimation $66 \%$ of the measured damping for the point $\mathrm{p}_{3}$ simulations in all of the microslip regime. Besides these cases, impulsive excitations at other points produce damping curves that are closer to the modal Iwan model for these two modes. The modal Iwan model generally underestimates the measured damping for the most dominant modal signals by an average of $40 \%$ at large microslip amplitudes, and this error decreases to $25 \%$ at lower amplitudes. In macroslip, $\zeta_{r, M}$ generally overestimates those measured by an average of $60 \%$ in the seventh and eighth modes.

As seen for the three-mass system, $\zeta_{r, M}$ in late microslip regime for modes 7 and 8 underpredicts by an order of magnitude the damping measured from simulations transitioning from macroslip to microslip. In these simulations, the amplitude of the physical response was such that one or more of the physical Iwan elements entered macroslip, hence the measured damping reflects that of the peak region in the early macroslip regime. When the response was filtered into its component modes, however, the amplitude of each modal signal was such that its corresponding modal Iwan model predicts damping in the late microslip regime. It is because the measured damping for macroslip was attributed to the modal amplitude suggesting microslip that there resulted an order-magnitude of difference between the measured and predicted damping. As explained in the final paragraph of Section 3.1.3, joints are generally designed to maintain their integrity and remain well within the microslip regime, so it is not likely that this region of amplitude would be reached in practice.

In contrast to the seventh and eighth modes, the damping of modal Iwan model for the ninth mode is in considerable disagreement with those of simulations (up to $300 \%$ underestimation in microslip). This, in addition to the curves with the largest deviations in modes 7 and 8, are again accounted for by other significant modes in the response. Looking specifically at the results of simulation 6 in Table D.2 (third smallest excitation of point $\mathrm{p}_{1}$ in microslip), it can be seen from Fig. 15 that the ninth modal coordinate decays rapidly compared to the seventh and eighth coordinates, which decay at about the same rate. As discussed earlier, this increases the measured damping in mode 9 such that it deviates further from the modal Iwan model as time advances. However, one should note that the ninth mode is weakly excited from this input, so its contribution to the response may not be important. The damping for the seventh and eighth modes also show noticeable deviations, and both of these modes are important to the response. However, overall the modal Iwan model is still very appealing because it does a good job of capturing the damping in the system, both qualitatively and quantitatively, yet it is far simpler and more efficient to use than the fully coupled, nonlinear model. 


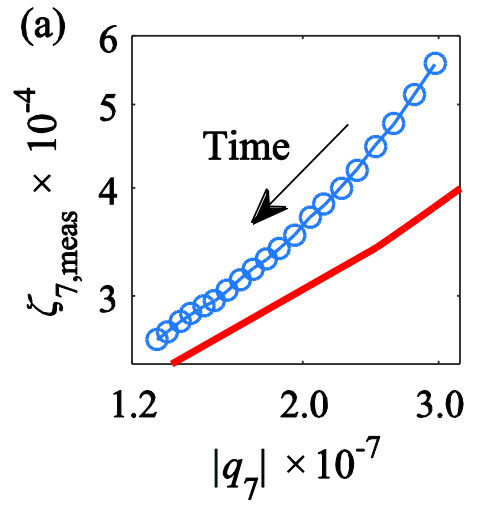

(b)

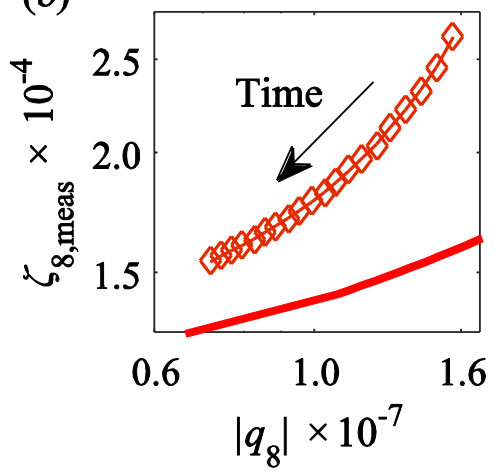

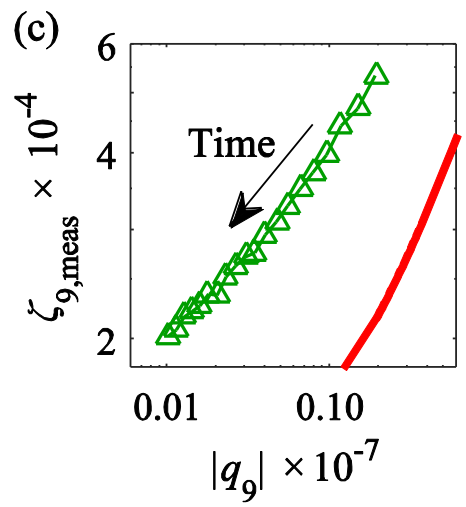

(d)

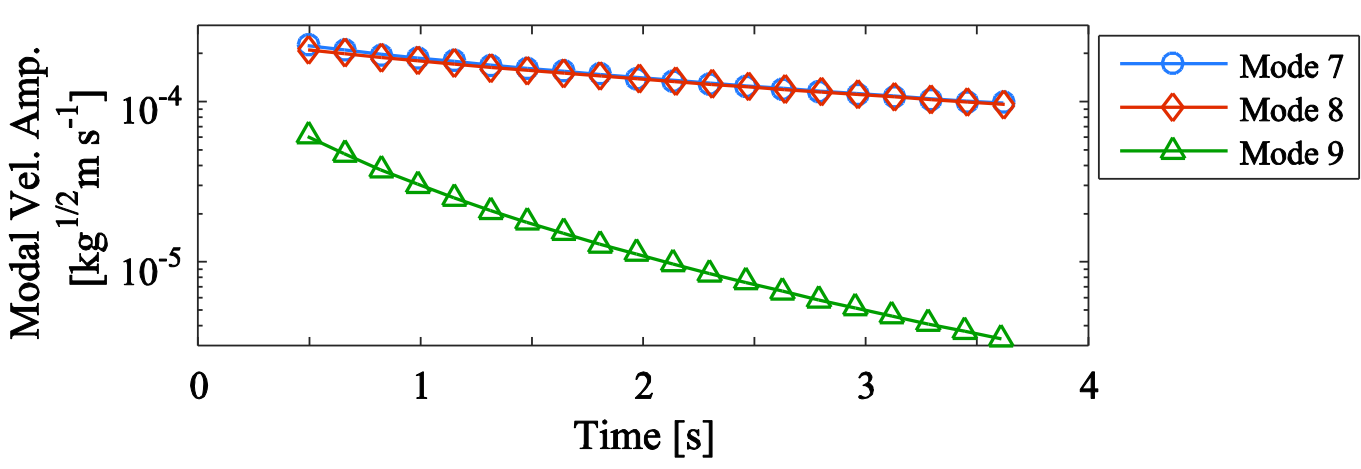

Fig. 15. Damping versus displacement amplitude with ideal curves in (a) mode 7, (b) mode 8, and (c) mode 9 response to an impulse in the Sumali beam (shown with $\zeta_{r, M}$ as the thick curve), and (d) time histories of the velocity amplitude for the three modes. The data are from simulation 6 in Table D.2. Displacement amplitude units are $\mathrm{kg}^{1 / 2} \mathrm{~m}$.

\section{Conclusion}

The key assumption of the modal Iwan model is that the modes of a structure are uncoupled, which allows each mode to be treated independently from others when calculating nonlinearity. If such an assumption is true, then it follows that a modal Iwan model can be tuned by selectively exciting each mode in turn and studying the change in the natural frequency and effective damping with amplitude. In a general response, however, all modes in a structure are excited to varying extent, so this work examined to what degree a modal Iwan model could still sufficiently capture the response of impulse-type excitations. Two numerical models - a threemass system and the Sumali beam finite element model - were studied to identify differences in the amplitude-dependent damping and natural frequency when the modes of the structure were selectively-excited, as compared to when the structure was excited with impulses at discrete points.

The results show that the modal Iwan models reproduce the effective natural frequency and damping of the response to impulsive excitations quite well provided that mode is dominant in the response. For the two systems studied in this work, the effective damping produced by the modal Iwan model accurately fits that of the measured response if the measured response contained one dominant mode (as by selective excitation). If more than one mode was dominant, 
then the damping predicted by modal Iwan models for the two systems typically exhibited less than $40 \%$ error in microslip and less than $60 \%$ error in macroslip. This error increases and can become as large as $400 \%$ if a mode is not dominant in the response, although in that case the response of such mode can probably be neglected. In many applications, these errors may be acceptable considering the computational savings and easy parameter identification that the modal Iwan model provides.

The alternative is to use a different nonlinear model that accounts for coupling between the modes, such as one developed by Festjens, Chavallier, and Dion [18]. In this case, one would have to assess whether the increase in computational cost from a coupled model is worth relinquishing the simplicity of uncoupled modal Iwan models, which in themselves still account for the change in modal damping and natural frequency with amplitude. Furthermore, to identify the parameters of such a model is not a trivial task because there are typically more unknown quantities than available data. Until a systematic methodology is developed by which a model can identified that includes modal coupling, the modal Iwan approach may be the best available alternative.

\section{Acknowledgements}

This work was funded by Sandia National Laboratories. Sandia National Laboratories is a multi-program laboratory managed and operated by Sandia Corporation, a wholly owned subsidiary of Lockheed Martin Corporation, for the U.S. Department of Energy's National Nuclear Security Administration under contract DE-AC04-94AL85000.

\section{Appendix A. Overview of the Four-Parameter Iwan Model}

In general, an Iwan element [10] is a one-dimensional, parallel arrangement of elastoplastic (Jenkins) elements as seen in Fig. A.1. These elastoplastic elements consist of a spring, all having uniform stiffness, in series with a friction slider having slider displacement $x$ and a certain slip displacement threshold $\phi$. If the $\phi$ for all elastoplastic elements is the same, then all sliders slip at the same Iwan element deflection $u$, and the Iwan element can be modeled equivalently by a single elastoplastic element. By setting different values of $\phi$ for each element, then the individual sliders slip one-by-one as $u$ deflects more, causing the Iwan element to lose stiffness in a piecewise manner until macroslip. In the limit that an Iwan element contains an infinite number of elastoplastic elements, the force-deflection curve, and by extension the hysteresis, of the Iwan element can be modeled continuously by assigning a population distribution function, $\rho(\phi)$, to define the relative proportion of sliders that slip at $\phi$. 
Fig. A.1. Schematic of the parallel-series Iwan model. Individual elastoplastic elements have the same spring stiffness but have a unique displacement threshold $\phi_{i}$ at which the contact (shown with a triangle) begins to slide.

Segalman's formulation defines $\rho$ as a truncated power-law terminated with a Dirac delta function of strength $S$ at the threshold of macroslip, $\phi_{\max }$,

$$
\rho(\phi)=R \phi^{\chi}\left[H(\phi)-H\left(\phi-\phi_{\max }\right)\right]+S \delta\left(\phi-\phi_{\max }\right),
$$

where $H(\cdot)$ is the Heaviside step function [11]. A graphical representation of this function is shown in Fig. A.2(a). Segalman then derives the force exerted by the Iwan element as

$$
\begin{aligned}
F_{I}(u(t)) & =\int_{0}^{\infty} \rho(\phi)[u(t)-x(t, \phi)] d \phi \\
& =\int_{0}^{\phi_{\max }} R \phi^{\chi}[u(t)-x(t, \phi)] d \phi+S\left[u(t)-x\left(t, \phi_{\max }\right)\right]
\end{aligned}
$$

Although this constitutive function was formulated using a continuous distribution of an infinite number of elastoplastic sliders, a numerical implementation of Eq. (A.2) is evaluated by tracking $x$ for a finite number of elements. 200 sliders were assigned to all Iwan elements in the simulations in this work to approximate a smooth force-deflection curve.

The four parameters, $R, S, \phi_{\max }$, and $\chi$, can be converted to more physically meaningful parameters, $F_{S}, K_{T}, \chi$, and $\beta$. Here, $F_{S}$ refers to the minimum force required to initiate macroslip, $K_{T}$ is the tangent stiffness of the joint, $\chi$ is the exponent of the power-law term, and $\beta$ is the ratio of the Dirac delta strength (related to macroslip) to the power-law strength [11],

$$
\begin{gathered}
F_{S}=\frac{R \phi_{\max }^{\chi+2}}{\chi+2}+S \phi_{\max }, \\
K_{T}=\frac{R \phi_{\max }^{\chi+1}}{\chi+1}+S, \\
\beta=\frac{S(\chi+1)}{R \phi_{\max }^{\chi+1}} .
\end{gathered}
$$

The reader is referred to [11] for a more rigorous treatment of the four-parameter Iwan model, but there are a few important ideas that should be emphasized in this brief treatment. Each elastoplastic element in an Iwan element can be thought of as representing a microscopic region of slip within the joint interface, and the gradual accumulation of sliders that pass the threshold of slip as the Iwan element flexes gives rise to microslip behavior. Equation (A.1) was chosen so as to induce power-law energy dissipation (and power-law damping, by extension) 
with respect to amplitude, a phenomenon that has been observed in several structures with metalon-metal joints $[12,16,17]$. The parameter $\chi$ can be observed directly through this relationship, as Segalman derived a log-log slope of $\chi+3$ in the microslip regime [11], as seen in Fig A.2(b).

(a)

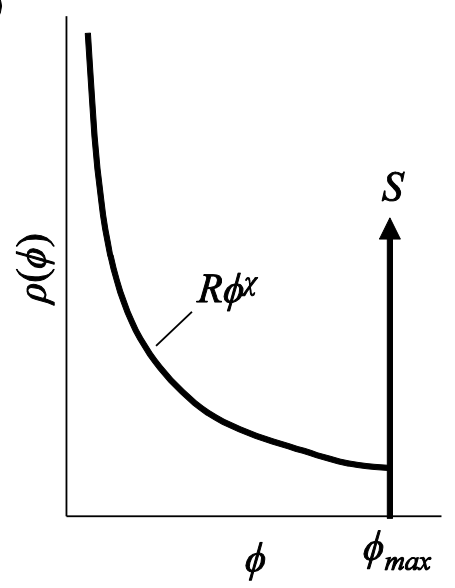

(b)

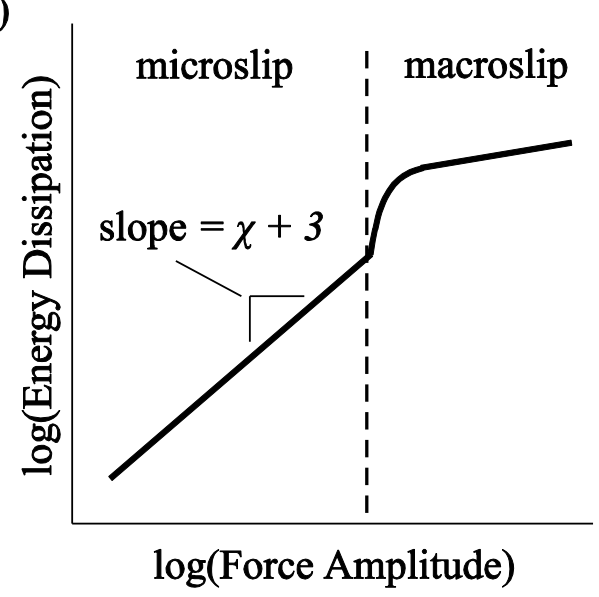

Fig. A.2. (a) The population distribution function of slider strengths associated with the fourparameter Iwan model, and (b) the resulting log-log energy dissipation per cycle for that model.

When all sliders have passed the threshold for slip (that is, when the last slider defined with $\phi_{\max }$ reaches its threshold), then the Iwan element enters macroslip, the model for which is equivalent to Coulomb friction. Experimentally, it is difficult to observe macroslip behavior from the response to an impact on a structure, so the Coulomb model for macroslip in cyclic loading has not yet been verified for impulse-type excitations. Despite this, the finite element models studied in this work all used four-parameter Iwan elements to represent physical joints, so the modal Iwan model was expected to capture their macroslip behavior to a good level of accuracy.

\section{Appendix B. Derivation of Equivalent Modal Damping from Energy Dissipation per Cycle}

While previous works focused on energy dissipation, which is a dimensional quantity, the authors found that it is convenient to express the effects of dissipation as an equivalent damping ratio for the mode in question. An expression for equivalent damping can be calculated from energy dissipation per cycle using the approach explained by Deaner et al. [16]. Consider the free response of a certain mode and presume that the amplitude decays very little (e.g. very light damping) within one response cycle. Then the velocity coordinate governing a particular massnormalized mode shape within that cycle can be expressed in an average sense over time $t$ within that single cycle as

$$
\dot{q}(t)=|\dot{q}| \sin \left(\omega_{d} t+\theta\right)
$$

where $|\dot{q}|$ is the modal velocity amplitude, $\omega_{d}$ is the damped natural frequency, and $\theta$ is an arbitrary phase. The energy dissipated within one cycle is then

$$
D=\int_{t}^{t+\frac{2 \pi}{\omega_{d}}} F_{v}(\tau) \dot{q}(\tau) d \tau
$$


If all sources of dissipation for this mode are approximated with an equivalent linear viscous damper, then the viscous damping force is $F_{v}(t)=C \dot{q}(t)=2 \zeta \omega_{n} \dot{q}(t)$, where $\omega_{n}$ is the undamped natural frequency and $\zeta$ is the equivalent modal damping ratio. Substituting this equation for $F_{v}$ in Eq. (B.2) and evaluating the integral gives

$$
D=2 \pi \zeta \frac{\omega_{n}}{\omega_{d}}|\dot{q}|^{2}=2 \pi \frac{\zeta}{\sqrt{1-\zeta^{2}}}|\dot{q}|^{2},
$$

while noting that the definition $\omega_{d}=\omega_{n} \sqrt{1-\zeta^{2}}$ from linear vibration theory is used. Equation (B.3) is dimensionally correct only when $\dot{q}(t)$ governs a mass-normalized mode shape; the quantity $|\dot{q}|^{2}$ is scaled correctly as such.

Rearranging Eq. (B.3), and extending the equation for any arbitrary cycle in a free response with instantaneous amplitude $|\dot{q}(t)|$, the effective damping ratio can be expressed over all time as

$$
\zeta(t)=\frac{D(t)}{\sqrt{4 \pi^{2}|\dot{q}(t)|^{4}+(D(t))^{2}}} .
$$

For lightly damped modes, the kinetic energy quantity $4 \pi^{2}|\dot{q}(t)|^{4}>>(D(t))^{2}$, so Eq. (B.4) can be simplified to

$$
\zeta(t)=\frac{D(t)}{2 \pi|\dot{q}(t)|^{2}} .
$$

It may also be convenient to express $\zeta(t)$ in terms of displacement amplitude $|q(t)|$ or acceleration amplitude $|\ddot{q}(t)|$. Equation (B.1) may be differentiated or anti-differentiated with respect to $t$ to obtain a relationship between these quantities and the velocity amplitude,

$$
\omega_{d}(t)|q(t)|=|\dot{q}(t)|=\frac{|\ddot{q}(t)|}{\omega_{d}(t)} .
$$

\section{Appendix C. Amplitude plots for the three-mass system}

Figure C.1 originates from the analysis in Section 3.1.1, and it shows the modal Iwan model analytical fits to the simulations that selectively excite modes 1 and 3 each at a time. 

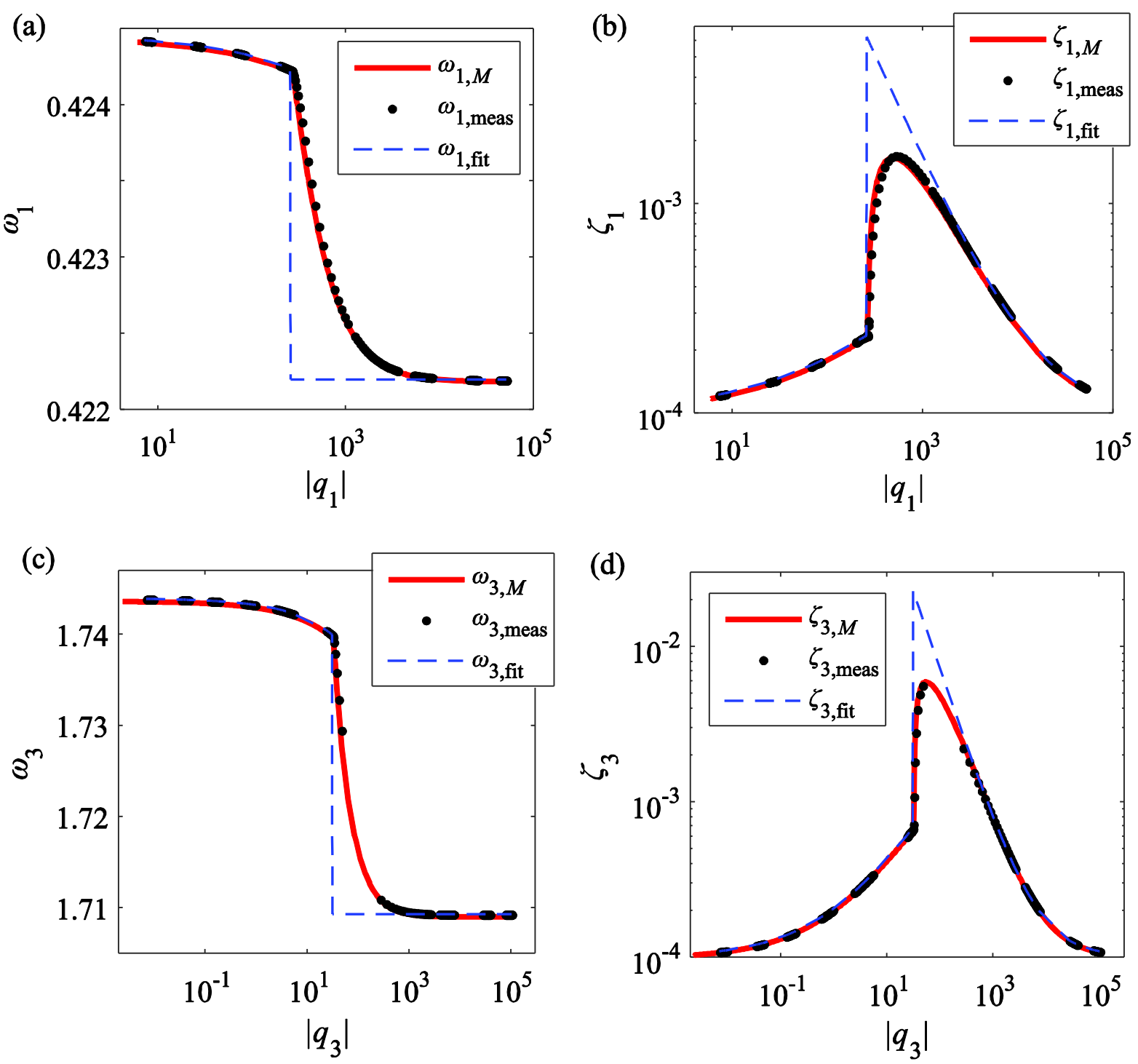

Fig. C.1. (a,c) Natural frequency and (b,d) critical damping ratio versus displacement amplitude for selective excitation of $(a, b)$ mode 1 and $(c, d)$ mode 3 in the three-mass system.

\section{Appendix D. Simulation parameters and amplitude plots for the Sumali beam system}

The frequency and damping versus amplitude curves for the modal Iwan models of the seventh and ninth modes in the Sumali beam finite element model are shown below. Table D.1 lists the simulation parameters for the mode shape excitation simulations in Section 3.2.2. The frequency and damping curves for modes 7 and 9 are plotted and fitted in Fig. D.1. Table D.2 lists the parameters for the discrete impulse simulations from Section 3.2.3. 
Table D.1

Parameters for the mode shape excitation simulations on the Sumali beam system. Scalar $p$ and vector $\varphi$ are the force multiplier value and mode shape, respectively, substituted into Eq. (27).

\begin{tabular}{|c|c|c|c|c|}
\hline $\begin{array}{l}\text { Simulation } \\
\text { Number }\end{array}$ & $p\left[\mathrm{~N} \mathrm{~kg}^{-1 / 2}\right]$ & $\varphi$ & Time Step [s] & Number of Steps \\
\hline 1 & 3361 & $\boldsymbol{\varphi}_{\infty 7}$ & $1 \times 10^{-5}$ & 200000 \\
\hline 2 & 1681 & $\boldsymbol{\varphi}_{\infty 7}$ & $5 \times 10^{-6}$ & 200000 \\
\hline 3 & 1008 & $\boldsymbol{\varphi}_{\infty 7}$ & $5 \times 10^{-6}$ & 200000 \\
\hline 4 & 336.1 & $\boldsymbol{\varphi}_{\infty 7}$ & $5 \times 10^{-6}$ & 100000 \\
\hline 5 & 67.23 & $\varphi_{\infty 7}$ & $5 \times 10^{-6}$ & 50000 \\
\hline 6 & 2.756 & $\boldsymbol{\varphi}_{07}$ & $5 \times 10^{-6}$ & 200000 \\
\hline 7 & 0.3361 & $\varphi_{07}$ & $3 \times 10^{-5}$ & 133333 \\
\hline 8 & 0.03361 & $\boldsymbol{\varphi}_{07}$ & $5 \times 10^{-5}$ & 200000 \\
\hline 9 & 10080 & $\boldsymbol{\varphi}_{\infty 88}$ & $1 \times 10^{-5}$ & 200000 \\
\hline 10 & 3361 & $\boldsymbol{\varphi}_{\infty 88}$ & $1 \times 10^{-5}$ & 100000 \\
\hline 11 & 1681 & $\boldsymbol{\varphi}_{\infty 88}$ & $5 \times 10^{-6}$ & 200000 \\
\hline 12 & 336.1 & $\boldsymbol{\varphi}_{\infty 8}$ & $5 \times 10^{-6}$ & 68000 \\
\hline 13 & 67.23 & $\boldsymbol{\varphi}_{\infty 8}$ & $5 \times 10^{-6}$ & 50000 \\
\hline 14 & 10.08 & $\boldsymbol{\varphi}_{08}$ & $8 \times 10^{-6}$ & 100000 \\
\hline 15 & 1.681 & $\boldsymbol{\varphi}_{08}$ & $1 \times 10^{-5}$ & 200000 \\
\hline 16 & 1.008 & $\boldsymbol{\varphi}_{08}$ & $4.7 \times 10^{-5}$ & 106383 \\
\hline 17 & 0.06723 & $\boldsymbol{\varphi}_{08}$ & $4.7 \times 10^{-5}$ & 212766 \\
\hline 18 & 26890 & $\varphi_{\infty 9}$ & $3 \times 10^{-6}$ & 333333 \\
\hline 19 & 6723 & $\boldsymbol{\varphi}_{\infty 9}$ & $3 \times 10^{-6}$ & 166667 \\
\hline 20 & 1681 & $\boldsymbol{\varphi}_{\infty 9}$ & $3 \times 10^{-6}$ & 69333 \\
\hline 21 & 67.23 & $\boldsymbol{\varphi}_{\infty 9}$ & $5 \times 10^{-6}$ & 20000 \\
\hline 22 & 3.361 & $\boldsymbol{\varphi}_{09}$ & $1 \times 10^{-5}$ & 100000 \\
\hline 23 & 0.2689 & $\boldsymbol{\varphi}_{09}$ & $1 \times 10^{-5}$ & 100000 \\
\hline 24 & 0.06723 & $\boldsymbol{\varphi}_{09}$ & $1 \times 10^{-5}$ & 200000 \\
\hline
\end{tabular}



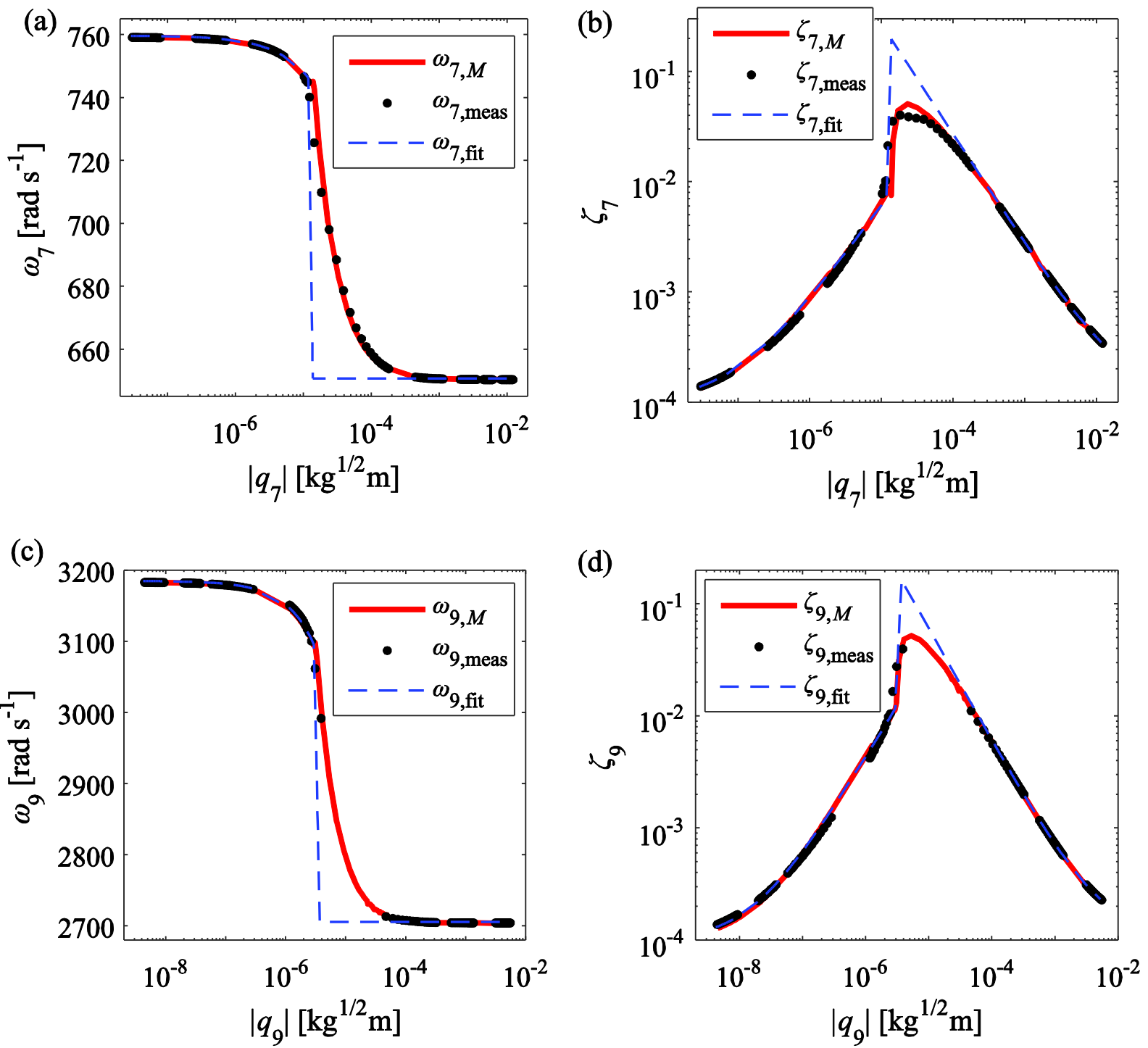

Fig. D.1. (a,c) Natural frequency and (b,d) critical damping ratio versus modal displacement amplitude for selective excitation of $(a, b)$ mode 7 and $(c, d)$ mode 9 in the Sumali beam system. 


\section{Table D.2.}

Parameters for the discrete impulse simulations on the Sumali beam system. The excitation point refers to the location in Fig. 9 where the impulse is applied. Scalar $p$ is the force multiplier value input to Eq. (29).

\begin{tabular}{|c|c|c|c|c|}
\hline Sim. No. & $p[\mathrm{~N}]$ & Time Step [s] & Number of Steps & Excitation Point \\
\hline 1 & 1779 & $3 \times 10^{-6}$ & 333333 & \multirow{8}{*}{$\mathrm{p}_{1}$} \\
\hline 2 & 889.6 & $3 \times 10^{-6}$ & 333333 & \\
\hline 3 & 133.5 & $5 \times 10^{-6}$ & 100000 & \\
\hline 4 & 8.896 & $1 \times 10^{-5}$ & 200000 & \\
\hline 5 & 1.335 & $1.3 \times 10^{-5}$ & 153846 & \\
\hline 6 & 0.2669 & $3 \times 10^{-5}$ & 133333 & \\
\hline 7 & 0.04448 & $5 \times 10^{-5}$ & 80000 & \\
\hline 8 & 0.01335 & $5 \times 10^{-5}$ & 160000 & \\
\hline 9 & 3114 & $5 \times 10^{-6}$ & 200000 & \multirow{10}{*}{$\mathrm{p}_{2}$} \\
\hline 10 & 889.6 & $5 \times 10^{-6}$ & 76000 & \\
\hline 11 & 133.5 & $5 \times 10^{-6}$ & 40000 & \\
\hline 12 & 26.69 & $5 \times 10^{-6}$ & 100000 & \\
\hline 13 & 4.448 & $1 \times 10^{-5}$ & 100000 & \\
\hline 14 & 1.335 & $1 \times 10^{-5}$ & 200000 & \\
\hline 15 & 0.4448 & $3 \times 10^{-5}$ & 133333 & \\
\hline 16 & 0.1335 & $5 \times 10^{-5}$ & 100000 & \\
\hline 17 & 0.04448 & $5 \times 10^{-5}$ & 100000 & \\
\hline 18 & 0.01335 & $5 \times 10^{-5}$ & 200000 & \\
\hline 19 & 4003 & $5 \times 10^{-6}$ & 200000 & \multirow{8}{*}{$\mathrm{p}_{3}$} \\
\hline 20 & 1334 & $5 \times 10^{-6}$ & 100000 & \\
\hline 21 & 222.4 & $5 \times 10^{-6}$ & 40000 & \\
\hline 22 & 22.24 & $5 \times 10^{-6}$ & 100000 & \\
\hline 23 & 8.896 & $1 \times 10^{-5}$ & 100000 & \\
\hline 24 & 3.559 & $2 \times 10^{-5}$ & 100000 & \\
\hline 25 & 0.8896 & $3 \times 10^{-5}$ & 100000 & \\
\hline 26 & 0.2224 & $5 \times 10^{-5}$ & 200000 & \\
\hline
\end{tabular}

\section{References}

[1] L. Gaul, J. Lenz, Nonlinear dynamics of structures assembled by bolted joints, Acta Mechanica, 125 (1997) 169-181.

[2] E.E. Ungar, The status of engineering knowledge concerning the damping of built-up structures, Journal of Sound and Vibration, 26 (1973) 141-154.

[3] D.J. Segalman, Modelling joint friction in structural dynamics, Structural Control and Health Monitoring, 13 (2006) 430-453. 
[4] E. Berger, Friction modeling for dynamic system simulation, Applied Mechanics Reviews, 55 (2002) 535-577.

[5] S. Bograd, P. Reuss, A. Schmidt, L. Gaul, M. Mayer, Modeling the dynamics of mechanical joints, Mechanical Systems and Signal Processing, 25 (2011) 2801-2826.

[6] E.P. Petrov, D.J. Ewins, Analytical formulation of friction interface elements for analysis of nonlinear multi-harmonic vibrations of bladed disks, Journal of Tubomachinery, 125 (2003) 364-371.

[7] B. Bhushan, Principles and Applications of Tribology, John Wiley \& Sons, 2013.

[8] K.L. Johnson, Contact Mechanics, Cambridge University Press, 1985.

[9] D.J. Segalman, An initial overview of Iwan modeling for mechanical joints, Sandia National Laboratories, Albuquerque, New Mexico, 2001, SAND2001-0811.

[10] W.D. Iwan, A distributed-element model for hysteresis and its steady-state dynamic response, Journal of Applied Mechanics, 33 (1966) 893-900.

[11] D.J. Segalman, A four-parameter Iwan model for lap-type joints, Journal of Applied Mechanics, 72 (2005) 752-760.

[12] D.J. Segalman, D.L. Gregory, M.J. Starr, B.R. Resor, M.D. Jew, J.P. Lauffer, N.M. Ames, Handbook on Dynamics of Jointed Structures, Sandia National Laboratories, Albuquerque, New Mexico, 2009, SAND2009-4164.

[13] D.J. Segalman, A modal approach to modeling spatially distributed vibration energy dissipation, Sandia National Laboratories, Albuquerque, New Mexico, 2010, SAND2010-4763.

[14] M. Feldman, Hilbert transform in vibration analysis, Mechanical Systems and Signal Processing, 25 (2011) 735-802.

[15] M. Eriten, M. Kurt, G. Luo, D.M. McFarland, L.A. Bergman, A.F. Vakakis, Nonlinear system identification of frictional effects in a beam with a bolted joint connection, Mechanical Systems and Signal Processing, 39 (2013) 245-264.

[16] B.J. Deaner, M.S. Allen, M.J. Starr, D.J. Segalman, H. Sumali, Application of viscous and Iwan modal damping models to experimental measurements from bolted structures, Journal of Vibration and Acoustics, 137 (2015) 021012 (021012 pages).

[17] D.R. Roettgen, M.S. Allen, Nonlinear characterization of a bolted, industrial structure using a modal framework, Mechanical Systems and Signal Processing, (2016).

[18] H. Festjens, G. Chevallier, J.L. Dion, Nonlinear model order reduction of jointed structures for dynamic analysis, Journal of Sound and Vibration, 333 (2014) 2100-2113.

[19] M. Feldman, Non-linear system vibration analysis using Hilbert transform--I. free vibration analysis method 'FREEVIB', Mechanical systems and Signal Processing, 8 (1994) 119-127. 
[20] H. Sumali, R.A. Kellogg, Calculating damping from ring-down using Hilbert transform and curve fitting, in: 4th International Operational Modal Analysis Conference (IOMAC), Istanbul, Turkey, 2011.

[21] M.S. Allen, H. Sumali, D.S. Epp, Piecewise-linear restoring force surfaces for seminonparametric identification of nonlinear systems, Nonlinear Dynamics, 54 (2008) 123135.

[22] R.D. Cook, D.S. Malkus, M.E. Plesha, R.J. Witt, Concepts and Applications of Finite Element Analysis, 4th ed., John Wiley \& Sons, Inc., New York, 2002.

[23] G. Reese, D. Segalman, M.K. Bhardwaj, K. Alvin, B. Driessen, K. Pierson, T. Walsh, C. Dohrmann, C.R. Wislon, Salinas-User's Notes, Sandia National Laboratories, Albuquerque, New Mexico, 2012, SAND99-2801.

[24] R.R. Craig, M.C.C. Bampton, Coupling of substructures for dynamic analyses, AIAA Journal, 6 (1968) 1313-1319. 